Technische Hochschule Wildau

$+$

Brandenburgische Technische UniversitätCottbus-

Senftenberg

Innovation Hub 13

fast track to

TR A N S F E R

Working Paper № 003

2021

Digitale Plattform für den Innovation Hub 13

Thomas Jacob 
fast track to transfer - die Working Paper Series des Innovation Hub 13 ermöglicht es Projektmitarbeiter:innen und Kooperationspartner:innen sowie weiteren an der Thematik des Wissens- und Technologietransfers interessierten Wissenschaftler:innen und Praktiker:innen, ihre Forschungsergebnisse und praktischen Erfahrungen der wissenschaftlichen Community sowie einer breiten Öffentlichkeit zur Diskussion zu stellen. Als Instrument zum "fast track to transfer" bietet sie die Möglichkeit, Ergebnisse, Einblicke und Erkenntnisse schnell zu veröffentlichen. Darüber hinaus soll sie den Diskurs mit Akteur:innen aus Wissenschaft, Wirtschaft, Verwaltung, Kultur und Kunst sowie der Zivilgesellschaft fördern, der Information dienen und dazu einladen, sich in die Diskussion um die Zukunft des Wissens- und Technologietransfers einzubringen.

$\underline{\text { www.innohub13.de/workingpaperseries }}$

Zitationsvorschlag:

Jacob, Thomas (2021): Digitale Plattform für den Innovation Hub 13.

Fast track to transfer (working paper series), No. 003,

DOI: 10.15771/innohub_3

Das Dokument erscheint unter der Creative-Commons-Lizenz

Namensnennung 4.0 International (CC BY 4.0)

\section{fast track to \\ T R A N S F E R}

Working Paper Series

Technische Hochschule Wildau Hochschulring 1

15745 Wildau

www.th-wildau.de

Brandenburgische Technische Universität Cottbus-Senftenberg

Platz der Deutschen Einheit 1

03046 Cottbus

www.b-tu.de

Weitere Informationen zum Innovation Hub 13 und zu Transfer finden Sie unter www.innohub13.de

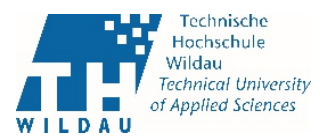

Brandenburgische Technische Universitä Cottbus - Senftenberg

Der "Innovation Hub 13 - fast track to transfer" der Technischen Hochschule Wildau und der Brandenburgischen Technischen Universität Cottbus-Senftenberg gehört zu den 29 ausgewählten Gewinnern der Bund-LänderFörderinitiative „Innovative Hochschule”, ausgestattet mit Mitteln des Bundesministeriums für Bildung und Forschung BMBF und des Landes Brandenburg. Weitere Informationen finden Sie unter www.innovative-hochschule.de

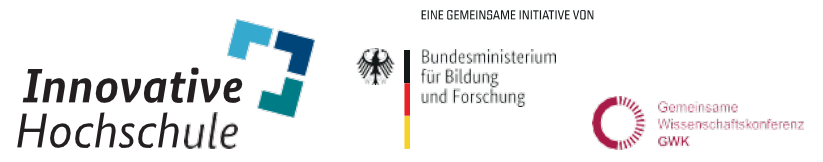




\title{
Digitale Plattform für den Innovation Hub 13
}

Jacob, Thomas, Technische Hochschule Wildau, „Innovation Hub 13 - fast track to transfer", thomas.jacob@th-wildau.de

\begin{abstract}
Das institutsübergreifende Transferprojekt Innovation Hub 13 soll eine Schnittstelle zwischen Wissenschaft, Wirtschaft und der Öffentlichkeit sein. Um dies zu realisieren, sollen digitale Werkzeuge und eine digitale Plattform den Transferscouts, die das Bindeglied zwischen den genannten Bereichen darstellen, eine Arbeitserleichterung verschaffen. Durch die Werkzeuge und die digitale Plattform sollen die Transferscouts kollaborativ mit Wissenschaft, Wirtschaft sowie Öffentlichkeit agieren und Kooperationen ermöglichen und in die Wege leiten. Eine systematische Verbreitung und Anwendung von wissenschaftlichem Know-how in die Projektregion ist dabei ein Ziel, was durch die digitale Plattform unterstützt werden kann. Zudem soll eine Öffnung der Hochschulen gegenüber der Zivilgesellschaft stattfinden sowie eine signifikante Verbesserung der Wissenschaftskommunikation erfolgen. Die Plattform soll dabei den gesamten Innovation Hub 13 digital abbilden. Das umfasst u.a. die Showrooms, die Testfelder und die Aktivitäten der Transferscouts. Dieses Working Paper zeigt auf, was eine digitale Plattform auszeichnet und welche Bestandteile diese beinhalten kann. Des Weiteren werden die konzeptionellen Arbeiten an der digitalen Plattform des Innovation Hub 13 dargelegt und einzelne Bausteine dieser digitalen Plattform beschrieben. Einige dieser Bausteine sind bereits umgesetzt und einzeln verwendbar. Zum Abschluss wird ein Ausblick über zukünftige Arbeiten an der konkreten digitalen Plattform des Innovation Hub 13 gegeben.
\end{abstract}

\section{Keywords}

Digitale Plattform, Innovation Hub 13, InnoRadar, InnoXR, VR, VueJS, Recommendation Engine 


\section{Inhaltsverzeichnis}

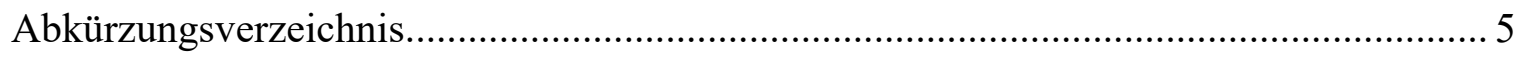

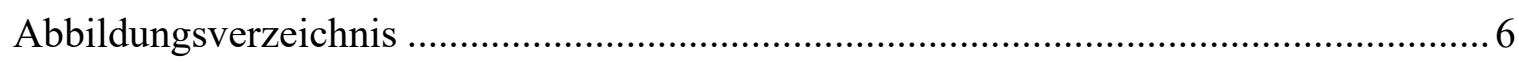

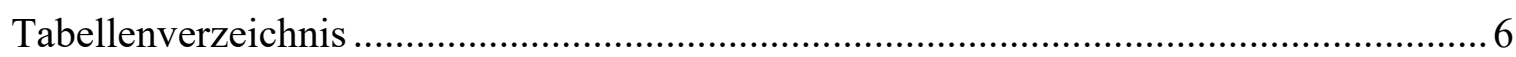

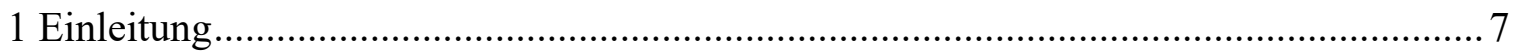

2 Definition digitale Plattform und Abgrenzung zu unterstützenden Softwarelösungen .... 8

3 Datenhaltung der digitalen Plattform des Innovation Hub 13 .................................... 9

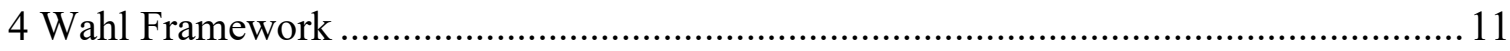

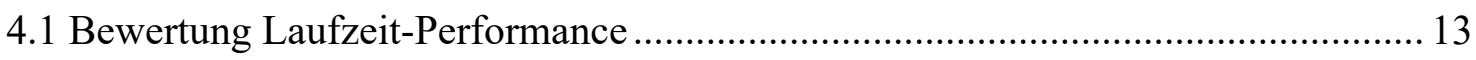

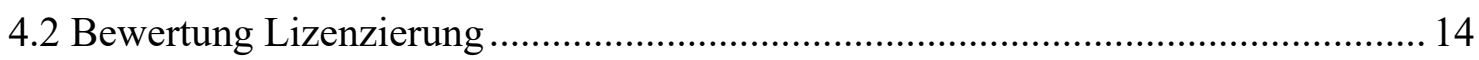

4.3 Bewertung Einfachheit/ Programmiersprachen ................................................. 14

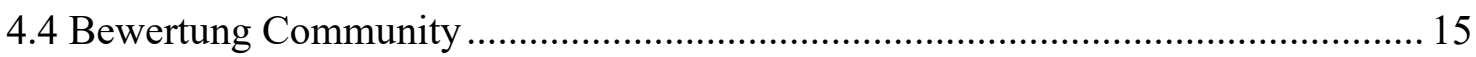

4.5 Auswertung der Bewertung und Auswahl des Frameworks .................................. 16

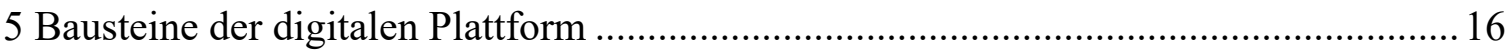

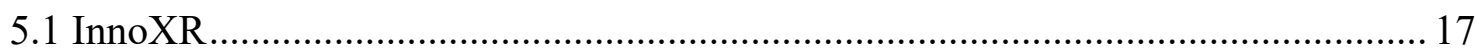

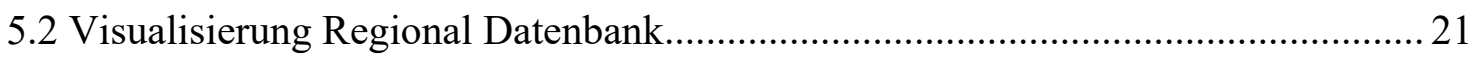

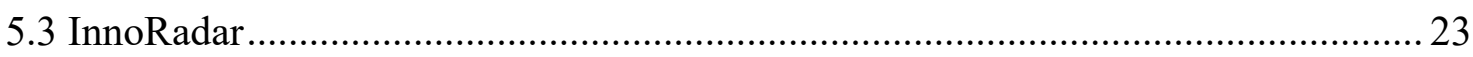

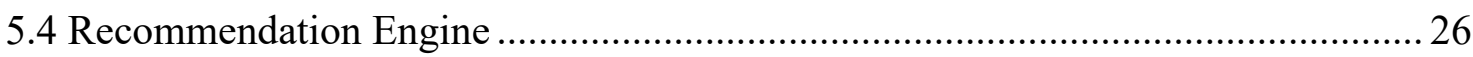

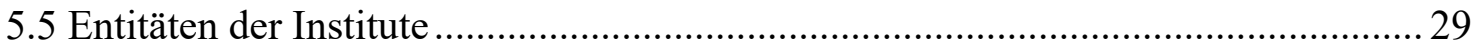

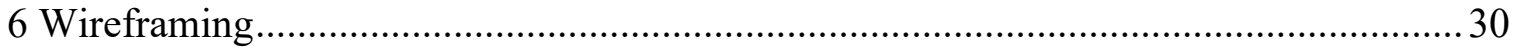

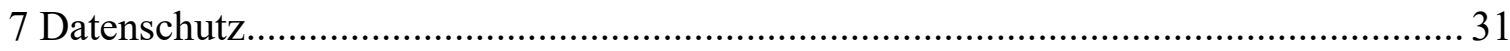

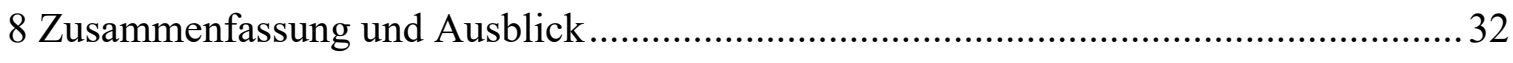

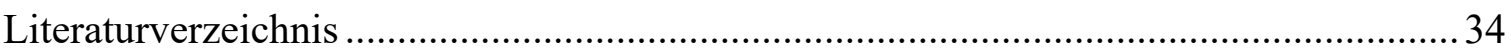




\section{$\underline{\text { Abkürzungsverzeichnis }}$}

\begin{tabular}{|c|c|c|}
\hline & API & Application Programming Interface \\
\hline & AR & Augmented Reality \\
\hline & CSS & Cascarding Style Sheets \\
\hline & $\mathrm{CSV}$ & Comma-separated values \\
\hline & DB & Datenbank \\
\hline & DBMS & Datenbankmanagementsystem \\
\hline & DSVGO & Datenschutz-Grundverordnung \\
\hline & ERM & Entity Relationship Modell \\
\hline$x$ & e.V. & Eingetragener Verein \\
\hline & $\mathrm{GmbH}$ & Gesellschaft mit beschränkter Haftung \\
\hline & GPS & Global Positioning System \\
\hline$\tilde{8}$ & HIT & Hochschulinformationstag \\
\hline$\stackrel{2}{2}$ & HTML & Hypertext Markup Language \\
\hline$\propto$ & IoT & Internet of Things \\
\hline 岁 & JS & JavaScript \\
\hline $\mathbf{z}$ & KDSF & Kerndatensatz Forschung \\
\hline & $\mathrm{M} 2 \mathrm{M}$ & Machine to Machine \\
\hline & MIT & Massachusetts Institute of Technology \\
\hline \pm & MQTT & Message Queuing Telemetry Transport \\
\hline & MR & Mixed Reality \\
\hline 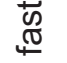 & MVC & Model-view-controller \\
\hline & PHP & Rekursives Akronym für PHP: Hypertext Preprocessor \\
\hline X & PoI & Point of Interest \\
\hline & RoR & Ruby on Rails \\
\hline & SSL & Secure Sockets Layer \\
\hline & TCP/IP & Transmission Control Protocol/Internet Protocol \\
\hline & TRL & Technology Readiness Level \\
\hline & TSB & Transfersteckbriefe \\
\hline & $\mathrm{TH}$ & Technische Hochschule \\
\hline & UX & User Experience \\
\hline & ViNN:Lab & Venture Innovation Lab \\
\hline & VR & Virtual Reality \\
\hline & $\mathrm{XR}$ & Extended Reality \\
\hline
\end{tabular}




\section{$\underline{\text { Abbildungsverzeichnis }}$}

Abbildung 1: Erstelltes ER-Modell für die Transferlösung im Innovation Hub .............. 13 mithilfe von AtriFlow …......................................................................... 10

Abbildung 2: Vergleich Ladezeiten-Metriken Frontend-Frameworks (Krause 2020) ...... 13

Abbildung 3: Screenshot InnoXR - VR Rundgang im ViNN:Lab .................................. 19

Abbildung 4: VR-Tour erstellt im Teilprojekt „Testbed“ ............................................... 20

Abbildung 5: Screenshot Stand der Arbeit Visualisierung Regional DB (Colv 2020) ..... 23

Abbildung 6: Screenshot InnoRadar für den Bereich Life Sciences

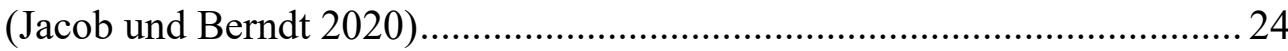

Abbildung 7: Einteilung Technology Readiness Level in drei Level

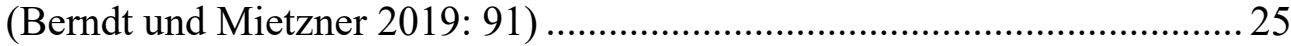

Abbildung 8: Screenshot Alpha-Version Recommendation Engine Startseite

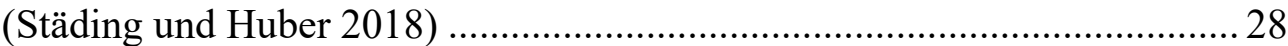

Abbildung 9: Prototypische Darstellung der Ergebnisse der Recommendation Engine

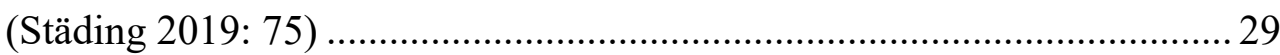

Abbildung 10: Wireframe-Entwurf der Startseite der digitalen Plattform ....................... 30

\section{Tabellenverzeichnis}

Tabelle 1: Kriterien für die Wahl des Frameworks für die Frontenderstellung der digitalen Plattform 10

Tabelle 2: Ergebnisse der Bewertung des Frameworks für die digitale Plattform ............ 14

Tabelle 3: Arbeitspakete der Visualisierung der Regional DB ........................................ 20 


\section{$\underline{1 \text { Einleitung }}$}

Im Innovation Hub 13 soll eine digitale Plattform bereitgestellt werden. Dabei ist angestrebt, mithilfe der Plattform die Arbeit der Transferscouts zu vereinfachen, eine Schnittstelle zu Unternehmen und Gesellschaft zu erzeugen sowie eine Verzahnung des virtuellen und physischen Innovation Hub 13 zu gewährleisten. Ziel der digitalen Plattform ist es, wissenschaftliches Know-how systematisch in der Region zu verbreiten und in die Anwendung zu bringen. Zudem soll eine Öffnung der Hochschulen gegenüber der Zivilgesellschaft stattfinden sowie eine Verbesserung der Wissenschaftskommunikation erreicht werden. Die digitale Plattform ist dabei ein Baustein des Innovation Hub $13 .{ }^{1}$ Weitere Bausteine sind u.a. Transferscouts, Testfelder und Showrooms, die nicht Bestandteil dieses Papers sind.

In diesem Working Paper wird erstens aufgezeigt, was unter einer digitalen Plattform zu verstehen ist und zweitens, wie der Stand der Arbeiten an der Plattform für den Innovation Hub 13 ist. Die digitale Plattform soll ein digitaler Zwilling des Innovation Hub 13 sein. Dazu sollen alle Bestandteile des Hubs, inkl. der Showrooms und Testfelder, als auch die Aktivitäten der Transferscouts, abgebildet werden.

Das Verständnis für die Definition einer digitalen Plattform ist im Laufe des Projektzeitraums gewachsen und hat sich von einer Bereitstellung von Softwarelösungen für die Mitarbeiter:innen des Innovation Hub 13 in die Zusammenführung der Kundengruppen auf der Plattform sowie die Darstellung eines digitalen Abbilds des Hubs gewandelt. Daher wird zunächst eine Definition einer digitalen Plattform gegeben und eine Abgrenzung dieser von den Werkzeugen dargestellt, die den Mitarbeiter:innen unterstützend zur Verfügung stehen, um ihre Arbeiten durchzuführen. Die Umsetzung der verwendeten Tools sind ebenfalls im Teilprojekt „Digitale Plattform“ verortet, aber sie gehören nicht zum hier vorgestellten Produkt der digitalen Plattform an sich.

Nachdem veranschaulicht ist, was unter einer digitalen Plattform zu verstehen ist, wird die geplante Datenhaltung der digitalen Plattform des Innovation Hub 13 beschrieben. Genauer wird beschrieben, wie die Daten wo gespeichert werden. Die Wahl des Frameworks, welches für die Entwicklung der digitalen Plattform eingesetzt werden soll, wird im darauffolgenden Kapitel betrachtet und die konzeptionellen Überlegungen für das in diesem Fall praktikabelste Framework dargestellt. Es wurden bereits einige Elemente entwickelt, die in die digitale Plattform integriert werden sollen. Andere Bausteine sind in der Entwicklung oder in der Konzeption. All diese Elemente werden beschrieben, um einen Überblick über mögliche Funktionen zu geben.

\footnotetext{
${ }^{1}$ Das hochschulübergreifende Projekt Innovation Hub 13 setzt sich aus zehn Teilprojekten zusammen, die jeweils andere Bereiche fokussieren. Das Teilprojekt „Digitale Plattform“ ist eines dieser Teilprojekte und es beinhaltet die Erstellung einer digitalen Plattform sowie die Bereitstellung digitaler Werkzeuge als Unterstützung für die anderen Projektmitarbeiter:innen.
} 
Nachdem die grundlegenden Bausteine der digitalen Plattform beschrieben worden sind, wird in der Folge auf die Konzeption mithilfe von Wireframes eingegangen. Durch diese Methode soll ein erster Einblick in den Aufbau und die Funktionsweise der zu entwickelnden Plattform gegeben werden. Auch eine Betrachtung der datenschutzrechtlichen Zusammenhänge der Plattform wird gegeben. Durch die Verarbeitung von personenbezogenen Daten, die auf der digitalen Plattform präsentiert werden sollen, ist dieser Bereich wichtig und sollte genauer betrachtet werden. Abschließend wird eine Zusammenfassung gegeben und ein Ausblick auf die Zukunft der digitalen Plattform gewagt. Dabei wird auf zukünftige Arbeiten eingegangen, die auf Basis dieses Working Papers durchgeführt werden müssen.

\section{Definition digitale Plattform und Abgrenzung zu unterstützenden Softwarelösungen}

Märkte funktionieren mehr und mehr zweiseitig. Die sogenannten Two-Sided Markets bringen verschiedene Gruppen an einem Ort zusammen. Durch die Verbindung zweier oder mehrerer Kundengruppen auf einer Plattform wird ein Mehrwert für alle diese Gruppen generiert. Die zwischen den Gruppen stehende Plattform ist meist nur Vermittler und verdient an dieser Vermittlung (Rochet und Tirole 2006: 645 ff.). Aufgrund der Tendenz zu Two-Sided Markets rücken digitale Plattformen in das Zentrum der Marktstrukturen. Der Wandel zu Plattform-Märkten wird durch den stärkeren Grad der Digitalisierung angetrieben und klassische Pipeline-Märkte ${ }^{2}$ werden verdrängt. Beispielsweise kann das US-amerikanische Dienstleistungsunternehmen Uber genannt werden. Das Unternehmen bietet eine Plattform an, auf der die Gruppe der Fahrgäste mit der Gruppe der Fahrer zusammengebracht wird. Durch die Plattform wird der Pipeline-Markt des Taxigewerbes zum Teil verdrängt. Digitale Plattformen können somit als Vermittler von Produkten, Dienstleistungen oder Technologien definiert werden. Eine Plattform zeichnet sich durch die Zusammenführung von zwei oder mehr Kundengruppen aus und partizipiert an der Vermittlung. Die Kundengruppen wiederum profitieren durch die über die Plattform zustande gekommenen Kontakte und können somit neue Kunden akquirieren oder auf der anderen Seite Dienstleistungen bzw. Produkte in Anspruch nehmen. Das Prinzip kann auf die geplante digitale Plattform des Innovation Hub 13 überführt werden. Ein Hauptziel, welches durch die Plattform unterstützt werden soll, ist die Zusammenführung der Forschenden und der Unternehmen. Der Innovation Hub 13 ist der Vermittler zwischen den Beteiligten und partizipiert an Kooperationen, wie beispielsweise gemeinsamer Forschungsprojekte: ein klassischer zweiseitiger Markt, der mithilfe der digitalen Plattform und der anderen Instrumente des Innovation Hub 13 gekoppelt wird. Diese Definition und deren Verständnis ist während des Projektzeitraums gewachsen. Sie änderte sich von einer Bereitstellung von Softwarelösungen für die Mitarbeiter:innen des Innovation Hub 13 in

\footnotetext{
${ }^{2}$ Klassische Pipeline-Unternehmen arbeiten in einer linearen Wertschöpfungskette. Von den Rohmaterialien zum Endprodukt wird gefertigt und die Zielgruppe sind die Kunden. Die Branchen dieser Unternehmen werden Pipeline-Märkte genannt.
} 
die Zusammenführung der Kundengruppen auf der Plattform sowie in die Darstellung eines digitalen Abbilds des Hubs. Um ein Verständnis für die Entwicklung der bisherigen Arbeiten zu schaffen, wird der Wandel in der Folge dargestellt.

$\mathrm{Zu}$ Beginn des Teilprojektes „Digitale Plattform“ wurde eine gemeinsame, teilprojektübergreifende Mind-Map erstellt, die mögliche Funktionen der digitalen Plattform darstellt. Der Fokus lag zu diesem Zeitpunkt auf der Unterstützung der Arbeit für die Transferscouts. Für diese Mind-Map wurde vorab eine Umfrage durchgeführt, welche Funktionen von den Projektmitarbeiter:innen für besonders wichtig erachtet wurden. Bei dem Meeting wurden die Funktionen priorisiert und zusammengefasst: So wurde bestimmt, dass mit Ausnahme der Recommendation Engine ${ }^{3}$ keine Selbstentwicklung durchgeführt wird, sondern fertige Produkte verwendet werden. Viele der gewünschten Funktionen könnten durch eine Kollaborationsplattform abgedeckt werden. Als nächster Schritt wurden unterschiedliche Kollaborationsplattformen mittels aus den Bedarfen erstellten Use Cases verglichen und bewertet.

Nach ausführlichen Tests von Demos der nach Umsetzung der Use Cases bestbewerteten Lösungen wurde die Nutzung der Software EGroupware präferiert. Die Lösung ist anschließend in der freien Version installiert und von den Projektmitarbeiter:innen getestet worden. Alle weiteren Funktionen, die nicht von der Kollaborationsplattform abgebildet werden können, sollten sukzessive mithilfe anderer Tools umgesetzt und ergänzt werden. Die verwendeten Softwarelösungen und die genutzte Kollaborationsplattform sind nicht Bestandteil dieses Working Papers. Mit Ausnahme eines kurzen Exkurses in die Datenhaltung für die digitale Plattform im kommenden Kapitel werden diese nicht weiter betrachtet.

Das Produkt der digitalen Plattform soll sich auf die Darstellung der erhobenen Daten innerhalb des Innovation Hub 13 konzentrieren. Diese Daten sollen auf verschiedene Weisen dargestellt werden und den Nutzer:innen mithilfe von Such- und Filterfunktionen die richtigen Informationen geliefert werden. Da die geplante digitale Plattform datenlastig ist, ist die Haltung jener Daten von erhöhter Bedeutung.

\section{$\underline{3 \text { Datenhaltung der digitalen Plattform des Innovation Hub } 13}$}

Die Datenhaltung für die digitale Plattform soll zentral durchgeführt werden. Damit keine redundanten Datensätze entstehen, sollen die Daten aus bestehenden Systemen verwendet und maximal temporär zwischengespeichert werden. Als Hauptdatenquelle soll die Kollaborationsplattform dienen, welche angepasst an die Bedarfe vornehmlich der Transferscouts, die aufgenommenen Daten dieser beinhaltet. Nach Fertigstellung der Basis

\footnotetext{
${ }^{3}$ Eine Recommendation Engine oder auch Empfehlungsmaschine ist ein System, das den Nutzer:innen Produkte, Dienstleistungen, Informationen vorschlägt, basierend auf der Analyse von Daten. Mehr dazu im Kapitel Recommendation Engine
} 
der Arbeit für die Transferscouts wurde festgelegt, dass EGroupware als Datenquelle für die Recommendation Engine und die digitale Plattform genutzt werden soll. Allerdings ist dieses Vorgehen obsolet, da im Laufe des Projekts beschlossen wurde, dass die Kollaborationssoftware EGroupware nicht weiter genutzt werden soll, da die Bedienung den Usability-Ansprüchen nicht genügt und fehlende Kontrollmöglichkeiten bei der Dateneingabe zu den qualitativ schwachen Datensätzen führten. Als alternative Lösung wurde die Software AtriFlow vom Entwickler AtriNeo gewählt, bei der das Datenschema und somit die Eingabemöglichkeiten flexibel konfigurierbar sind. Das ermöglicht eine Anpassung an die Bedarfe der Transferscouts. Zudem ist mit der Nutzung von definierten Listen eine Minimierung der Eingabeinkonsistenzen gegeben. Dies sollte zu einer erhöhten Qualität der Datensätze führen und die Nutzer:innen bei der Eingabe unterstützen. AtriFlow stellt eine API4 bereit, mit deren Hilfe die Daten abgerufen werden können. Somit kann der Ansatz der zentralen Speicherung in Verbindung mit einem Zugriff der digitalen Plattform weiterhin fortgeführt werden. Damit die neu zu erstellende Transferplattform AtriFlow den Bedarfen der Transferscouts genügt, musste ein vollständiges Datenschema erstellt werden, das als Basis für die Software dient. Dafür wurde im ersten Schritt ein vereinfachtes ER-Modell5 konzipiert, welches die Bedarfe der Transferscouts in Datenform darstellt. Die Abbildung 1 zeigt das erstellte ER-Model.

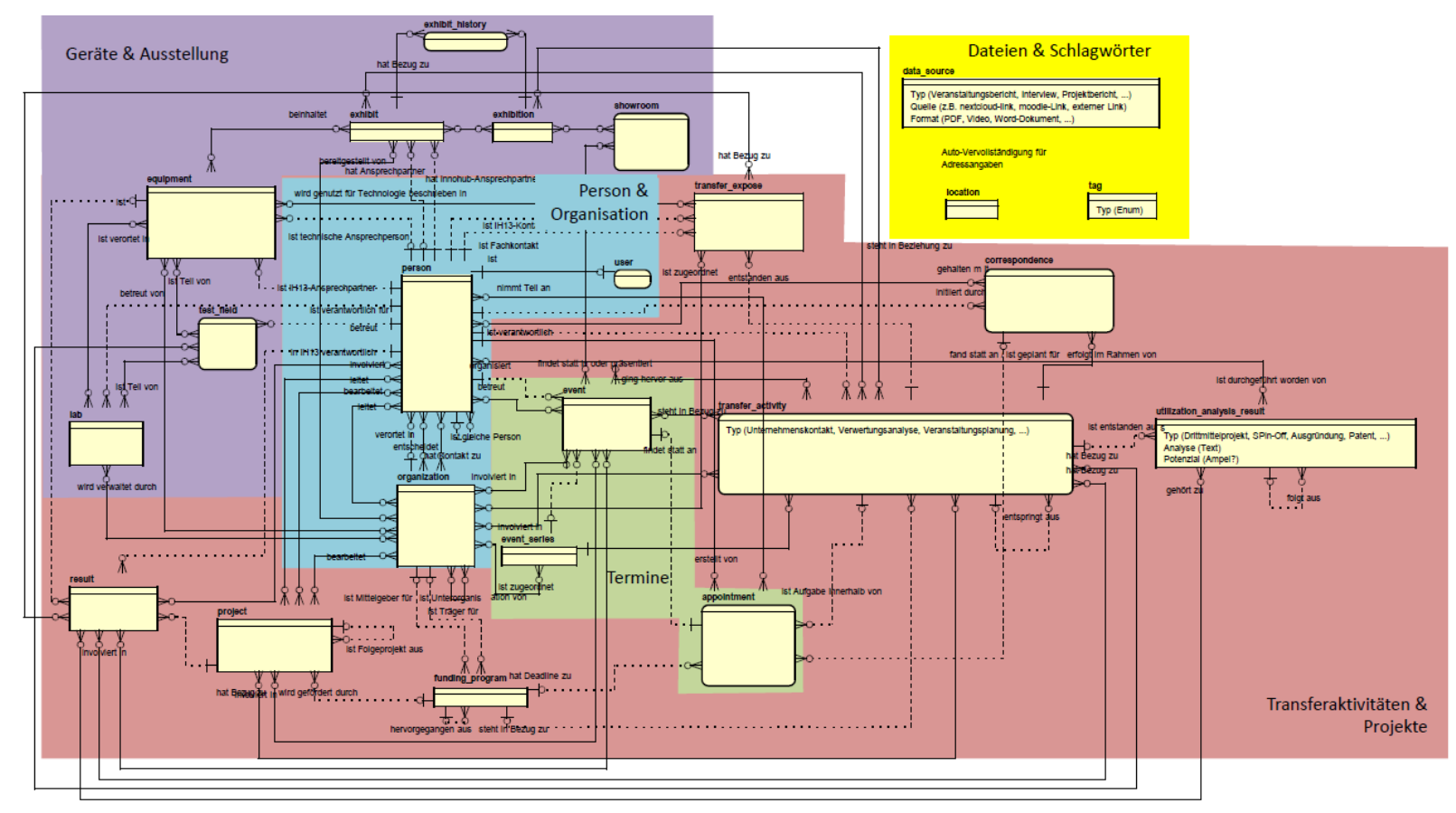

Abbildung 1: Erstelltes ER-Modell für die Transferlösung im Innovation Hub 13 mithilfe von AtriFlow.

\footnotetext{
${ }^{4}$ Application Programming Interface (API) ist eine Programmierschnittstelle, die auf Quelltext-Ebene angebunden wird.

${ }^{5}$ Ein Entity Relationship Modell (ERM) dient der Beschreibung von Daten in einem spezifischen Anwendungsbereich. Dabei stellt das ERM einen Ausschnitt der realen Welt dar und dient als Grundlage für den kommunikativen Austausch verschiedener Personen.
} 
Im ER-Modell sind die einzelnen Objekte (gelbe Kästen. Entitäten genannt.) und deren Zusammenhänge (Linien. Assoziationen genannt.) zu erkennen. Für eine bessere Übersichtlichkeit wurden die Entitäten in Gebiete aufgeteilt, welche die Kategorien darstellen und diese Gebiete farblich hinterlegt. Die Assoziationen unterscheiden sich in ihren Beziehungen zwischen zwei Entitäten. Dies wird durch die Verbinder an den Entitäten und die Linienform (gestrichelt oder durchgezogen) dargestellt. Die Assoziationen sind Bestandteil des zweiten Arbeitsschritts, welcher eine genaue Spezifikation der einzelnen Entitäten als Aufgabe hatte. Genauer wurden in diesem Schritt alle Entitäten im Fließtext beschrieben, genaue Attribute erstellt, die die Eigenschaften eines Objektes darstellen, und die Verbindungen zwischen den Entitäten festgelegt. Beide miteinander verbundenen Arbeitsschritte stellten eine Gesprächsgrundlage für die Umsetzung der ersten Version der AtriFlow-Lösung dar. Mit dem Erstellen einer ersten Version beginnt ein iterativer Prozess, welcher eine Weiterentwicklung der Transferlösung als Ziel hat. In Zusammenarbeit mit den Entwickler:innen der Software sollen die Anzeigen, Funktionen und Ausgaben so angepasst werden, dass alle Prozesse der Transferscouts abgebildet werden können. Die Nachverfolgung und Umsetzung aller Prozesse der Transferscouts ist demnach dann auf der Plattform intuitiv benutzbar. Nach Abschluss dieses iterativen Prozesses können die bisherigen Daten importiert werden. Dies führt zu einer Datenhaltungs-Basis, die sowohl für die Recommendation Engine als auch für die digitale Plattform nutzbar ist.

\section{Wahl Framework}

Auf dem Markt befindet sich eine Vielzahl an Frameworks, die für eine Erstellung von Frontends für Webanwendungen konzipiert sind. Die Wahl eines Frameworks ist auf Basis der Vielzahl der Optionen äußerst schwierig und abhängig von den subjektiven Vorlieben der Entwickler:innen sowie den Ansprüchen der zu entwickelnden Lösung. In diesem Kapitel wird versucht, objektiv ein Framework auszuwählen, welches die Entwicklung der digitalen Plattform ermöglicht und einen geringe Einarbeitungsschwelle besitzt. Bedingung ist die Basis JavaScript für die Frontend-Entwicklung. Für eine Vorauswahl der Frameworks wird die jährliche Studie JavaScript Rising Stars von Michael Rambeau verwendet. Diese Studie basiert auf den Bewertungen der Entwickler:innen auf GitHub ${ }^{6}$ der letzten zwölf Monate. Das Ergebnis der aktuellen Studie, in Bezug auf Frontend Frameworks, ist im Folgenden dargestellt (Rambeau 2020):

- Platz 1 Vue.js

- Platz 2 React

- Platz 3 Angular

\footnotetext{
${ }^{6}$ GitHub ist eine Plattform, auf der Programmcode verschiedener Projekte verwaltet wird. Die Plattform bietet eine einfache Versionsverwaltung und die Bearbeitung von Quellcode durch mehrere Entwickler:innen. Der Programmcode kann synchronisiert und verteilt werden.
} 
Beim Vergleich der Frameworks sind verschiedene Kriterien miteinzubeziehen. Dies sind Laufzeit-Performance, Lizenzierung, Programmiersprachen, Einfachheit in der Entwicklung und Community. Die Kriterien werden gewichtet und einzeln bewertet. Dadurch soll eine maximale Objektivität gegeben werden. Abgrenzend werden die Frameworks ausschließlich auf jene Kriterien in diesem Kapitel vorgestellt und nicht allgemein beschrieben. In der Tabelle 1 werden die Kriterien aufgezeigt, kurz beschrieben und deren Gewichtung genannt. Um aus den qualitativen Kriterien quantitative Bewertungen zu erhalten, wird eine Bewertungsskala von 0 bis 10 eingeführt. Die 10 steht dabei für das bestmögliche Ergebnis. Für die Endberechnung werden die einzelnen Bewertungen mit der jeweiligen Gewichtung des Kriteriums ${ }^{7}$ multipliziert und anschließend alle Werte eines Frameworks aufaddiert. Somit erhält jedes Framework eine Gesamtbewertung zwischen 0 und 10.

\begin{tabular}{|c|c|c|}
\hline Kriterium & Gewichtung & Kurzbeschreibung \\
\hline $\begin{array}{l}\text { Laufzeit- } \\
\text { Performance }\end{array}$ & $40 \%$ & $\begin{array}{l}\text { Im Zusammenhang mit der Usability einer Plattform ist die } \\
\text { Geschwindigkeit dieser essentiell. Dabei sind Lade- und } \\
\text { Bearbeitungsgeschwindigkeiten zu betrachten. Dieses Kriterium ist } \\
\text { für die digitale Plattform des Innovation Hub } 13 \text { besonders hoch } \\
\text { gewichtet, da die Nutzerzufriedenheit im Fokus steht. }\end{array}$ \\
\hline Lizenzierung & $20 \%$ & $\begin{array}{l}\text { Die Nutzung eines Frameworks muss rechtlich problemlos } \\
\text { funktionieren. Um dies sicherzustellen und um die Offenheit zu } \\
\text { gewährleisten, sind Lizenzen wichtig. Eine freie Nutzung ist } \\
\text { notwendig und ein offener Quellcode vorteilhaft. }\end{array}$ \\
\hline $\begin{array}{l}\text { Einfachheit/ } \\
\text { Programmier- } \\
\text { sprachen }\end{array}$ & $25 \%$ & $\begin{array}{l}\text { Alle gewählten Frameworks basieren auf JavaScript. Zusätzlich } \\
\text { werden HTML sowie CSS verwendet. Für den leichten Einstieg ist es } \\
\text { wichtig, dass genau diese Sprachen genutzt werden, da sie bei den } \\
\text { meisten Entwickler:innen bekannt sind und so die Einarbeitungszeit } \\
\text { verringert wird. Zusätzlich sind weitere Barrieren bei der Einführung } \\
\text { eines solchen Frameworks und auch die tendenzielle Lernkurve der } \\
\text { Entwickler:innen bei Erstbenutzung eines solchen Frameworks zu } \\
\text { beachten. }\end{array}$ \\
\hline Community & $15 \%$ & $\begin{array}{l}\text { Die Community ist aus zweierlei Hinsicht als Kriterium geeignet. } \\
\text { Zum einen ist es durch eine große Community wahrscheinlicher, dass } \\
\text { bei Problemen sowie Unklarheiten Lösungsansätze präsentiert } \\
\text { werden können, da sich viele Entwickler:innen in der Bedienung des } \\
\text { jeweiligen Frameworks tiefgreifend auskennen. Zum anderen ist die } \\
\text { Weiterentwicklung des Frameworks sichergestellt, da die } \\
\text { Entwicklung entweder direkt durch die Community funktioniert oder } \\
\text { alternativ das Entwicklerunternehmen durch eine aktive sowie große } \\
\text { Community dazu motiviert wird, das Framework weiterzuentwickeln. }\end{array}$ \\
\hline
\end{tabular}

Tabelle 1: Kriterien für die Wahl des Frameworks für die Frontenderstellung der digitalen Plattform.

\footnotetext{
${ }^{7}$ Wobei die Gewichtung von Prozent auf einen absoluten Zahlenwert zwischen 0 und 1 umgerechnet wird. Beispielsweise entspricht $10 \%$ einem Wert von 0,1 .
} 


\subsection{Bewertung Laufzeit-Performance}

Für die Bewertung der Laufzeit-Performance wird ein Benchmark von Stefan Krause verwendet, der verschiedene JavaScript-Frameworks regelmäßig testet und unter anderem die Ladegeschwindigkeit sowie die Speicherausnutzung diverser Frameworks vergleicht. In der Abbildung 2 sind die Ergebnisse übersichtlich zusammengefasst.

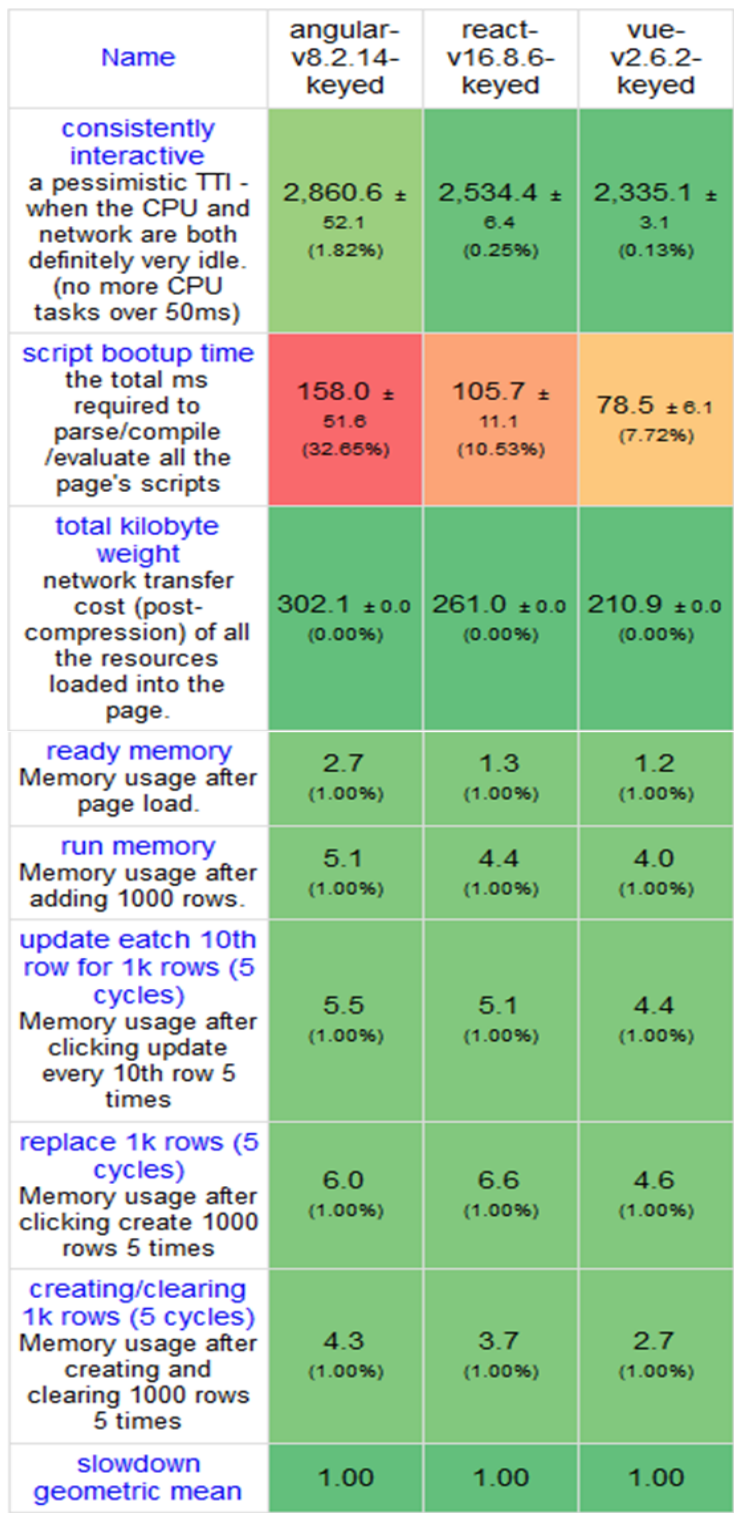

Abbildung 2: Vergleich Ladezeiten-Metriken Frontend-Frameworks (Krause 2020).

In der Abbildung ist zu erkennen, dass Vue.js durch seinen kleineren Rahmen bei der Ladegeschwindigkeit am besten abschneidet. React ist ebenfalls schnell bei der Startgeschwindigkeit, aber nicht auf dem Niveau von Vue.js. Angular ist aufgrund der umfangreichen Struktur vergleichsweise langsam beim Laden. Bei der Speicherzuweisung sind Vue.js und React ebenfalls nah beieinander, wobei Vue.js besser abschneidet. Angular benötigt deutlich mehr Speicher und ist daher langsamer als die anderen beiden 
Frameworks. Aufgrund der Ergebnisse des Benchmarks werden Angular 5, React 8 und Vue.js die vollen 10 Bewertungspunkte zugewiesen.

\subsection{Bewertung Lizenzierung}

Bei der Lizenzierung der einzelnen Frameworks für die Webseiten-Frontendentwicklung werden deren freie Nutzung und der offene Quellcode berücksichtigt.

Angular ist unter der MIT-Lizenz ${ }^{8}$ verfügbar und wird hauptsächlich von Google entwickelt. Eine MIT-Lizenz ist sehr positiv zu bewerten, da der Quellcode frei verfügbar ist und beliebig verwendet werden darf. Aufgrund dessen wird Angular mit 10 Punkten in dieser Kategorie bewertet.

React wurde von Facebook entwickelt und ist in der aktuellen Version MIT lizenziert. Damit ist der Quellcode frei verfügbar und nutzbar. Allerdings ist das Entwicklungsunternehmen Facebook eher schlecht zu bewerten, da dieses die Lizenz in der Vergangenheit mehrfach geändert hat (Rixecker 2017). Daher erhält React im Bereich Lizenzierung eine Bewertungspunktzahl von 7.

Vue.js wurde durch Evan You entwickelt und steht wie die anderen beiden Frameworks unter einer MIT-Lizenz. Daher erhält das Framework ebenfalls 10 Bewertungspunkte in der Kategorie Lizenzierung.

\subsection{Bewertung Einfachheit/ Programmiersprachen}

Die Einfachheit eines Frameworks ist äußerst subjektiv und daher schwierig quantitativ bewertbar. Um dies trotzdem $\mathrm{zu}$ ermöglichen, werden verschiedene Quellen zusammengetragen und deren Einschätzung zur Einfachheit und Lernkurve ausgewertet. Da bei Webentwickler:innen JavaScript, HTML sowie CSS als Programmier- bzw. Auszeichnungssprachen weit verbreitet sind, ist eine Verwendung dieser Sprachen essentiell. Eine Verwendung spezieller Programmiersprachen für einzelne Frameworks beeinflusst das Ergebnis negativ, da diese in vielen Fällen neu erlernt werden müssen und dies zu einer Verlangsamung der Erlernung führt. Eine ausführliche Dokumentation der Funktionen erleichtert die Anwendung eines Frameworks, da auf diese Weise die passenden Funktionen gefunden werden können und deren Nutzung beschrieben wird. Daher ist auch der Umfang der Dokumentation Bestandteil der Bewertung dieses Kriteriums.

Angular basiert auf JavaScript, aber das Framework wird mithilfe einer eigenen Programmiersprache namens TypeScript programmiert. Diese Programmiersprache beruht auf JavaScript und erweitert diese um die Verwendung von Objekten. Diese Objektorientierung unterscheidet sich von der JavaScript-Programmierung und sorgt für

\footnotetext{
${ }^{8}$ Die MIT-Lizenz ist eine aus dem Massachusetts Institute of Technology stammende Open-Source-Lizenz. Sie erlaubt die Wiederverwendung der unter ihr stehenden Software (Teklote 2019).
} 
eine Erschwerung bei der Einarbeitung in das Framework, wenn die oben genannten Programmiersprachen der Entwickler:innen bekannt sind. Generell gilt die Einarbeitungsphase in Angular als langwierig. Lernerfolge sind nur langsam zu erreichen. Die Dokumentation des Frameworks wird positiv erwähnt (Koenig 2019; Schlemmer 2019; Skoczylas o. J.; Tappert 2020; Teufel 2018). Aufgrund der Programmiersprache, die es zu erlernen gilt, und der langen Einarbeitungsphase, erhält Angular in dieser Kategorie den Bewertungspunktewert 2. React gilt vom Grundsystem her als leicht zu erlernen. Die Programmierung basiert auf JavaScript. Problematisch wird es, wenn über das Grundsystem hinaus Funktionen benötigt werden. Dann müssen die Entwickler:innen zusätzlichen Lernaufwand investieren. Die Dokumentation gilt auch bei React als sehr gut (Schlemmer 2019; Skoczylas o. J.; Springer 2018; Tappert 2020). Aufgrund der positiven Bewertungen der unterschiedlichen Quellen, erhält das Framework 9 Bewertungspunkte. Vue.js gilt bei den Entwickler:innen als leicht zu erlernen. Lernerfolge sind schnell zu erreichen. Durch den modularen Aufbau sind Erweiterungen einfach möglich und das Framework an die Bedürfnisse anzupassen. Vue.js basiert auf einfachem JavaScript. Die Dokumentation gilt durchweg als gut, wobei in Bezug auf die Dokumentation React und Angular etwas besser bewertet werden (Koenig 2019; Schlemmer 2019; Springer 2018; Tappert 2020; Teufel 2018). Durch die einfache Erlernbarkeit wird das Framework hoch bewertet. Nur die etwas schwächere Dokumentation führt zu einem Abzug von einem Bewertungspunkt. Somit erhält Vue.js in dieser Kategorie 9 Punkte.

\subsection{Bewertung Community}

Durch eine große Community ist es wahrscheinlicher, dass bei Problemen sowie Unklarheiten Lösungsansätze präsentiert werden können, da sich viele Entwickler:innen in der Bedienung des jeweiligen Frameworks tiefgreifend auskennen. Ebenso ist die Weiterentwicklung eines Frameworks sichergestellt, da die Entwicklung entweder direkt durch die Community funktioniert oder alternativ das Entwicklungsunternehmen durch eine aktive sowie große Community dazu motiviert wird, das Framework weiterzuentwickeln. Aus diesen Gründen ist es für ein Framework wichtig, eine große und aktive Community zu besitzen. Daher ist die Community eine Kategorie bei der Bewertung der einzelnen Frameworks. Für die Bewertung wird auch das Entwicklungsteam miteinbezogen.

Hinter Angular steht Google, wodurch ein großer finanzieller Spielraum und ein großes Entwicklungsteam gegeben sind. Zudem pflegt eine imposante Open Source Community das Framework und erweitert es kontinuierlich. Bei Problemstellungen kann die Community stets helfen. Daher erhält Angular 10 Bewertungspunkte in dieser Kategorie. React hat mit Facebook ebenfalls einen großen Internetkonzern im Hintergrund, welches sich in einer hohen finanziellen Unterstützung und einem großen Entwicklungsteam widerspiegelt. Zudem ist die Open Source Community immens gewachsen. React können daher ebenfalls 10 Bewertungspunkte in der Kategorie Community gegeben werden. 
Vue.js stammt ursprünglich von einem Entwickler und wurde von diesem vorangetrieben. Mittlerweile ist die Abhängigkeit von diesem Entwickler durch eine Erweiterung des Entwicklungsteams verringert und eine finanzielle Unterstützung durch mehrere Unternehmen gegeben, aber sowohl die Teamgröße als auch die finanziellen Mittel sind geringer als bei den anderen beiden Frameworks. Die Open Source Community ist stark gewachsen, aber noch nicht einer Stufe mit React und Angular. Aus diesen Gründen erhält Vue.js eine Bewertung von 6 Punkten in diesem Kriterium.

\subsection{Auswertung der Bewertung und Auswahl des Frameworks}

In diesem Abschnitt werden die Ergebnisse der Bewertung der Frontend-Frameworks für die Entwicklung der digitalen Plattform zusammengefasst und eine Auswahl des passenden Frameworks durchgeführt. Die Tabelle 2 zeigt die Ergebnisse der Bewertung übersichtlich zusammengefasst.

\begin{tabular}{|l|c|c|c|c|}
\hline Kriterium & Gewichtung & Vue.js & React & Angular \\
\hline Laufzeit-Performance & 0,4 & 10 & 8 & 5 \\
\hline Lizensierung & 0,2 & 10 & 7 & 10 \\
\hline Einfachheit/ Programmiersprachen & 0,25 & 9 & 9 & 2 \\
\hline Community & 0,15 & 6 & 10 & 10 \\
\hline Gesamtbewertung & & $\mathbf{9 , 1 5}$ & $\mathbf{8 , 3 5}$ & $\mathbf{6 , 0 0}$ \\
\hline
\end{tabular}

Tabelle 2: Ergebnisse der Bewertung des Frameworks für die digitale Plattform.

In der Tabelle ist zu erkennen, dass das Framework Vue.js, mit Ausnahme der Bewertung für die Community, stets das beste Resultat erzielt hat. Das Framework erreicht eine Gesamtbewertung von 9,15 und ist somit deutlich besser bewertet als das zweitbeste Framework React mit einer Bewertung von 8,35. Mit deutlichem Abstand folgt Angular, welches vor allem aufgrund der weniger guten Laufzeit-Performance eine schlechtere Gesamtbewertung von 6,00 erhält.

Trotz der geringeren Community, die allerdings stark anwächst, ist die Wahl des Frameworks, basierend auf der Bewertung, eindeutig. Für die Erstellung der digitalen Plattform des Innovation Hub 13 wird daher Vue.js gewählt.

\section{Bausteine der digitalen Plattform}

Die digitale Plattform wurde bisher nur konzeptionell geplant und in einer einfachen ersten Version umgesetzt. Trotz dessen sind bereits Bausteine, die später essentiell für die Plattform sind, erstellt worden bzw. werden aktuell entwickelt. Die Bausteine stellen Inhalte dar, die in die digitale Plattform integriert werden müssen. Ausnahme bildet die Entitäten der Institute ${ }^{9}$, welche nur konzeptionell bedacht sind.

${ }^{9}$ Siehe Kapitel Entitäten der Institute 


\subsection{InnoXR}

Die digitale Plattform hat die Aufgabe, den Innovation Hub 13 digital abzubilden. Dazu gehören auch die Showrooms, Testfelder und ggf. die Labore der beteiligten Partnerinstitute. Da eine Visualisierung dieser Bestandteile des Innovation Hub 13 jenem Ziel dienlich ist, wurde InnoXR als virtuelles Branding erstellt, wobei $\mathrm{XR}^{10}$ für Extended Reality steht. Unter dem Branding InnoXR werden somit alle Ergebnisse der Bereiche VR, AR und MR zusammengefasst. Bisher lag der Fokus auf der virtuellen Realität, aber auch im Bereich Augmented Reality wurden erste prototypische Ergebnisse erzielt, die hier nicht weiter ausgeführt werden. InnoXR soll in Zukunft ein Bestandteil der digitalen Plattform sein und deren Angebot erweitern.

Für die digitale Präsentation der einzelnen Labore der beteiligten Partnerinstitute sind virtuelle Laborrundgänge erstellt worden. Das Angebot der Rundgänge wird stetig erweitert und somit werden fortwährend mehr Labore virtuell bereitgestellt. Dabei sind die VR-Rundgänge zum einen für die Nutzung am Computer ausgelegt, aber andererseits auch für die Betrachtung in speziellen VR-Brillen konzipiert.

Für die Erstellung der VR-Bilder wird eine spezielle Kamera eingesetzt. Die Insta360 Pro ist für die Erstellung hochauflösender $360^{\circ}$-Fotos konzipiert und nimmt durch die Verwendung von sechs versetzten Fotolinsen den gesamten Raum um die Kamera zeitgleich auf. Die Aufnahmen können ferngesteuert oder mithilfe eines Timers ausgelöst werden.

In einem ersten Versuch wurden prototypische VR-Rundgänge mit dem Open Source Framework A-Frame ${ }^{11}$ erstellt. Eine Darstellung der $360^{\circ}$-Bilder funktioniert gut. Durch Erweiterungen sind verschiedene Anbindungen von JavaScript und ähnlichem möglich. Aufgrund dessen ist ein flexibler Einsatz des Frameworks an die Bedarfe der jeweiligen Nutzer:innen möglich. Das größte Problem der Lösung ist die aufwändige Platzierung der Navigationspunkte und weiterer Points of Interest (PoI). Das führt zu einem großen Zeitaufwand für die Erstellung eines VR-Rundganges. Deshalb wurden Alternativen gesucht und mit der Software 3DVista Virtual Tour auch gefunden. Die Software ermöglicht die Erstellung einzelner Touren für die Labore. Die Software ermöglicht, einzelne VR-Rundgänge in Projekten zu erstellen und in diesen per Drag \& Drop sogenannte Hotspots zu platzieren. Diese Hotspots werden bei den erstellten VR-Touren für die Navigation und für die PoI genutzt. Die Navigation bietet die Möglichkeit, sich zwischen den unterschiedlichen Kamerapositionen innerhalb eines Labors zu bewegen.

\footnotetext{
${ }^{10}$ Extended Reality (XR) bezieht sich auf alle realen und virtuellen Umgebungen, die durch Computergrafik und Wearables erzeugt werden. XR ist der Oberbegriff für alle verschiedenen Formen der computerveränderten Realität, einschließlich der erweiterten Realität (Augmented Reality, AR), der gemischten Realität (Mixed Reality, MR) und der virtuellen Realität (Virtual Reality, VR) (Irvine 2017).

${ }^{11}$ A-Frame ist ein Web-Framework zum Aufbau von virtuellen Realitäten. A-Frame basiert auf HTML, benötigt keiner Installation und macht den Einstieg einfach. Das Framework ist nicht nur eine Auszeichnungssprache; der Kern ist ein leistungsstarkes Entity-Component-Framework, das eine deklarative, erweiterbare und zusammensetzbare Struktur bietet (A-Frame o. J.).
} 
Dabei werden weiße Pfeile genutzt, um einen Raumwechsel innerhalb des Labors durchzuführen (z.B. durch eine Tür) und weiße Kreise, um innerhalb desselben Raums zu navigieren. Die Points of Interest werden durch einen orangen Kreis mit einem kleinen weißen „i“ dargestellt. Bei einem Klick auf diesen Punkt wird ein Infofeld mit Bild- und Textteil geöffnet. Alternativ können Poster als Bild oder ein Video angezeigt werden. Es gibt noch weitere Optionen, wobei das Ausführen von JavaScript vorrangig die Möglichkeiten solcher Hotspots aufzeigt, da dadurch Änderungen vorgenommen, dynamisch Inhalte präsentiert oder ein Zugriff auf externe Quellen gegeben werden können. Die Software bietet die Möglichkeit, die fertigen VR-Touren in verschiedenen Formaten zu exportieren. Für die Verwendung auf einem Server kann eine Version exportiert werden, die auf HTML und JavaScript basiert. Diese Version kann nur mittels Internetbrowser von einem Server abgerufen werden und funktioniert nicht lokal auf einem Computer. ${ }^{12}$ Diese VR-Rundgänge funktionieren mit den gängigen Browsern. ${ }^{13}$ Alternativ können die VR-Touren auch als Standalone-Variante ausgegeben werden. Dabei kann je eine Version für Microsoft Windows und das Apple iOS exportiert werden. Dies hat den großen Vorteil, dass die VR-Rundgänge offline, also ohne Zugang zum Internet, ausgeführt werden können. Das ist für Messen, Präsentationen, Konferenzen usw. wichtig, da nicht immer sichergestellt ist, dass ein Internetzugang gegeben ist.

Mit der Software wurden mehrere prototypische VR-Rundgänge erstellt, wobei eine Zusammenstellung für die Nacht der kreativen Köpfe 2019 an der BTU CottbusSenftenberg, die aus zwei Laboren der BTU Cottbus-Senftenberg ${ }^{14}$, drei Laboren der TH Wildau $^{15}$ sowie die Campus in Cottbus und Senftenberg bestand, explizit zu nennen ist. Diese Tour war die erste öffentliche Präsentation. Obwohl der Rundgang prototypisch und medial nicht „state of the art“ war, fiel das Feedback der Besucher:innen der Nacht der kreativen Köpfe durchweg positiv aus. Dadurch wurde gezeigt, dass die Touren positive Effekte für den Innovation Hub 13 haben können und es ratsam erscheint, an diesen kontinuierlich weiterzuarbeiten.

So entstand in der Folge die prototypische Einzeltour für das CVision:Lab der TH Wildau, welche die erste vollständige Laborpräsentation des Innovation Hub 13 darstellt. Diese Einzeltour und fünf weitere wurden genutzt, um zur Wissenschaftswoche 2020 an der TH Wildau eine Präsentation der virtuellen Laborrundgänge, die durch den Innovation Hub 13 erstellt worden, durchzuführen. Im Detail waren dies die an der TH Wildau verorteten Labore CVision:Lab, Labor für Luftfahrttechnik, ViNN:Lab, Werkstofftechnik I, Werkstofftechnik II sowie die Präsenzstelle in Luckenwalde. Der Screenshot in Abbildung 3 zeigt den virtuellen Rundgang.

\footnotetext{
${ }^{12}$ Unter Vernachlässigung der Möglichkeit, lokal einen Server zu erstellen.

${ }^{13}$ Getestet mit Google Chrome, Mozilla Firefox und Microsoft Edge.

${ }^{14}$ Ausbildungslabor für Zellkulturtechnik und Zentrum effiziente Fabrik.

${ }^{15}$ Logistikwerkstatt, ViNN:Lab sowie Labor für Maschinendynamik und lärmarme Konstruktion.
} 


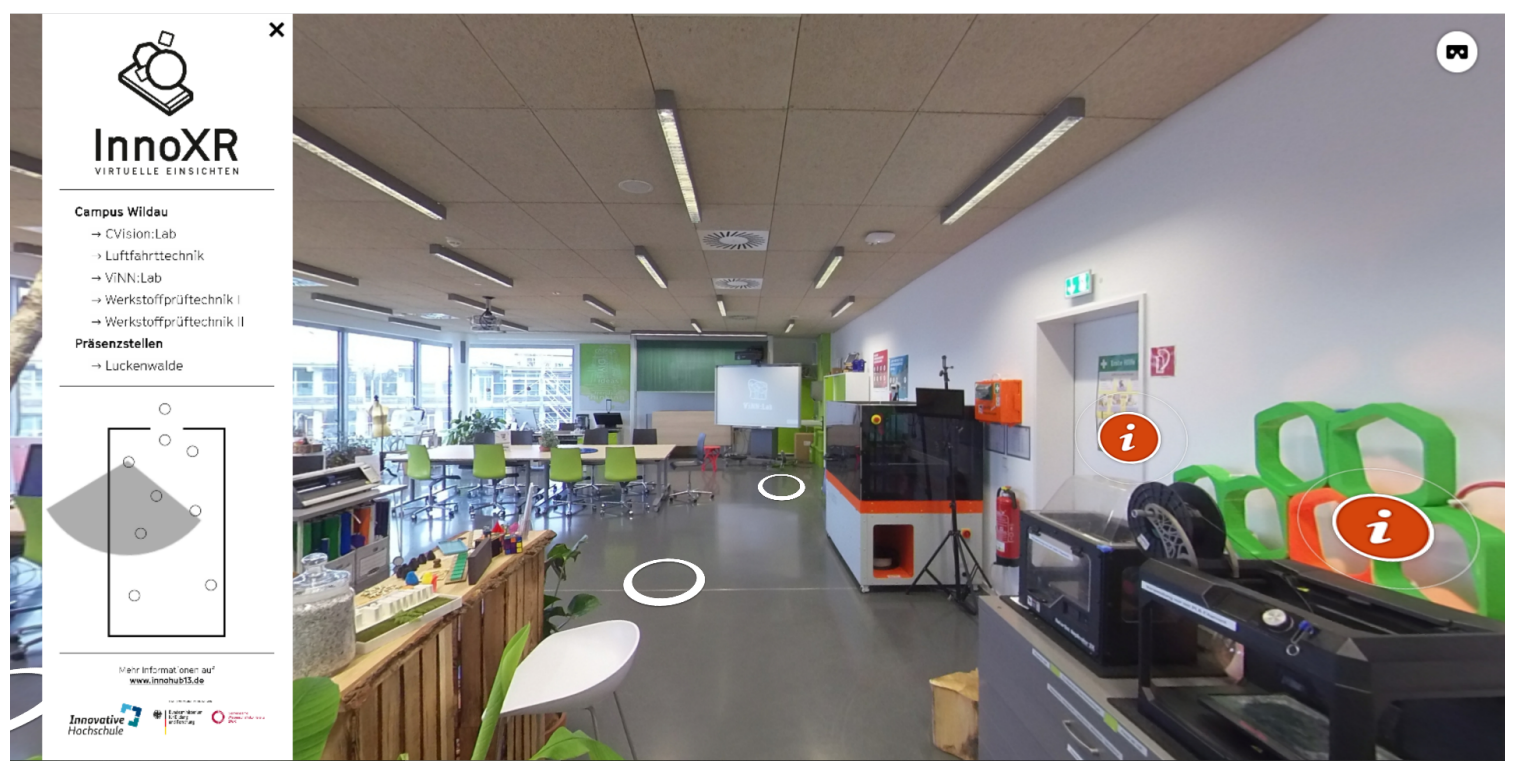

Abbildung 3: Screenshot InnoXR - VR Rundgang im ViNN:Lab.

Neben den bekannten Hotspots ist der VR-Tour-übergreifende Navigationsbereich im linken Bildsegment des Screenshots zu erkennen. Dieser Bereich beinhaltet einen virtuellen Lageplan des jeweiligen Labors inklusive eines Radars, was die Sichtrichtung der Nutzer:innen darstellt. Der Lageplan dient der Orientierung der Nutzer:innen und zusätzlich als Navigationsmöglichkeit, da die einzelnen Punkte auf die unterschiedlichen $360^{\circ}$-Aufnahmen verweisen. Oberhalb des Lageplans sind die vorhandenen Labore dargestellt. Durch einen Klick auf das jeweilige Labor wird zu diesem navigiert. Das ermöglicht eine laborübergreifende Navigation, trotz der Eigenständigkeit jedes Labors als ein Projekt. Diese Navigation funktioniert nur in der Serverversion und nicht in der Standalone-Variante, da in der Offline-Version die Software versucht, auf den Server zuzugreifen, wo die VR-Touren gehostet werden. Das Feedback auf der Wissenschaftswoche war durchgehend positiv.

Bei der Erstellung der virtuellen Rundgänge für die Labore wurden die Grenzen der Software deutlich. In der Folge werden kurz die größten Diskrepanzen von 3DVista Virtual Tour in Bezug auf die Bedürfnisse des Innovation Hub 13 aufgezeigt:

- Es wird durch die Software keine Unterstützung für Vektorgrafiken gegeben. Alle verwendeten Bilder sind pixelbasiert. Somit kann es bei besonders hochauflösenden Bildschirmen zu Darstellungsproblemen kommen. Die größte Auswirkung hat dies bei der Darstellung des Navigationsbereichs im linken Bildsegment, welches aus einem Bild besteht. Die Anpassung auf verschiedene Bildgrößen funktioniert nicht vollständig.

- Der linke Navigationsbereich ist, wie im ersten Punkt beschrieben, ein Bild. In diesem Bild werden Hotspots gesetzt: für die Verlinkung der anderen Labore, die laborinternen Verweise und den Link zur Homepage des Innovation Hub 13. Wenn 
ein neues Labor in diese laborübergreifende Tour integriert werden soll, muss in allen Laborprojekten das jeweilige Bild ausgetauscht werden. Anschließend müssen alle Hotspots neu gesetzt werden. Dies bedeutet einen hohen Arbeitsaufwand für die Integration neuer Labore.

- Die Software unterstützt einige Anzeigemöglichkeiten nicht in der VR-Brille. So können beispielsweise Informationsfelder und der Lageplan in der Brille aktuell nicht angezeigt werden (Stand: Mai 2020). Dadurch werden unterschiedliche Versionen für die VR-Brille und die Computeransicht erstellt. Dies hat verschiedene Folgen. Beispielhaft ist eine Navigation nur über Hotspots im Raum möglich. Das gilt für die laborinterne sowie -übergreifende Navigation. Die Informationsfelder werden als Bild eingebunden, welches vorher erstellt wird. Insgesamt bedeutet dies, dass zwei Versionen für einen virtuellen Rundgang gepflegt werden müssen, was einen erhöhten Arbeitsaufwand bedeutet.

- Eine weitere Grenze, die durch die Software gegeben ist, sind die mangelnden Gestaltungsmöglichkeiten der Informationsfelder. Diese ermöglichen keine Integration von HTML und eigenem CSS. Dadurch sind die gestalterischen Optionen beschränkt.

All diese Grenzen führten zu dem Bedarf einer verbesserten Lösung für die Umsetzung der virtuellen Rundgänge. Im Teilprojekt „Testbed“ des Innovation Hub 13 entsteht eine Präsentationsplattform für die Testfelder, die im Projekt erstellt werden. In dieser Plattform soll eine Möglichkeit gegeben werden, VR-Touren darzustellen und einzupflegen. Dabei soll eine übergreifende Navigation, eine flexible Erstellung von Informationsfeldern und eine einfache Integration von Hotspots möglich sein. Dies führt dazu, dass, sobald die Funktionsweise nachgewiesen ist, die vorhandenen VR-Rundgänge dort erneut erstellt und alle folgenden ebenso in dieser Plattform umgesetzt werden. Ab diesem Zeitpunkt ist 3DVista Virtual Tour obsolet. Der Screenshot in Abbildung 4 zeigt die aktuelle Version (Stand Juni 2021) der VR-Touren, die über die Testfeldplattform erstellt werden.

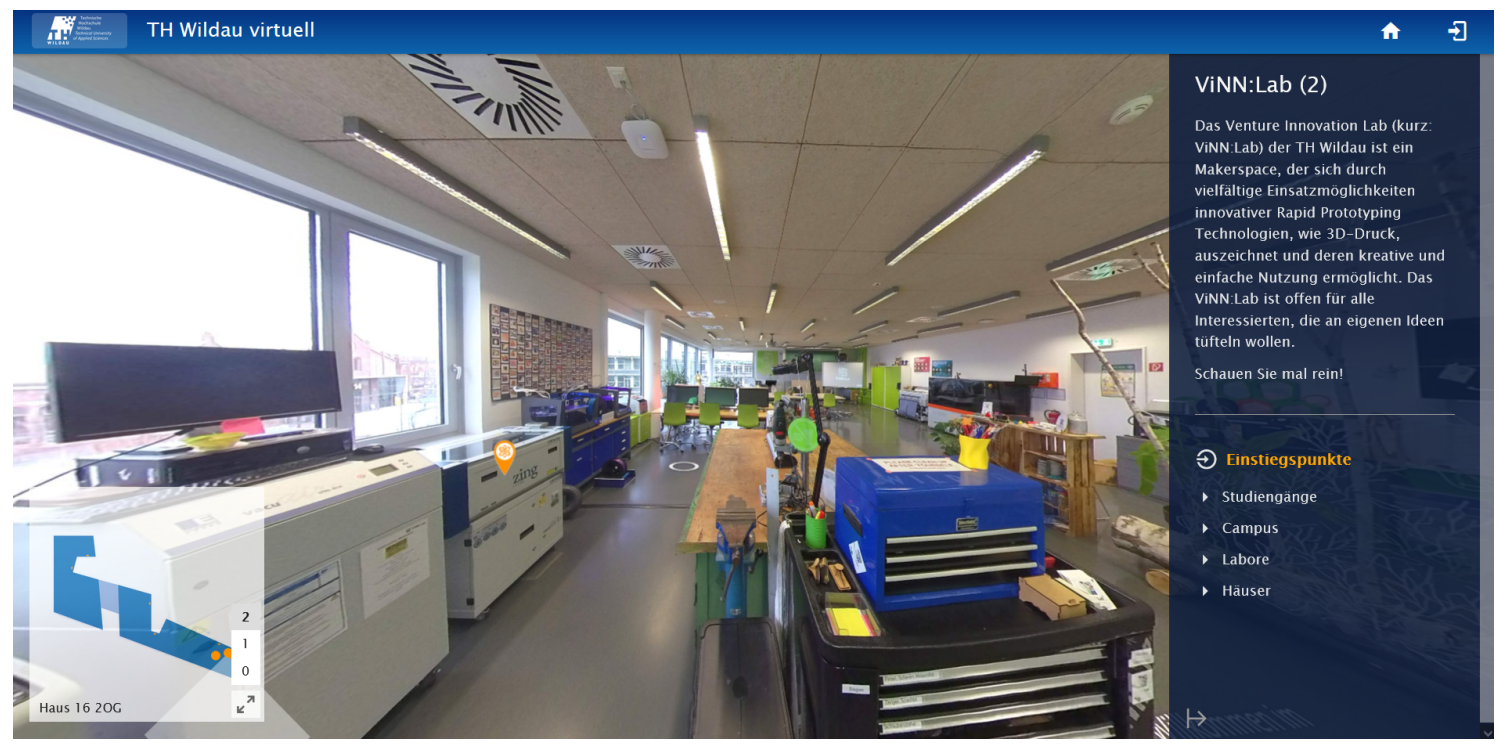

Abbildung 4: VR-Tour erstellt im Teilprojekt „Testbed“. 
Diese Version ist erstellt worden für den digitalen Hochschulinformationstag (HIT) an der TH Wildau. Auf dem Screenshot ist zu erkennen, dass PoIs gesetzt und gefiltert werden können. Zudem ist eine Navigation über Links in der Sidebar sowie über integrierte Navigationspunkte im Raum möglich. Nach aktuellem Stand ist davon auszugehen, dass diese Lösung zeitnah die bisherigen VR-Touren ablösen wird.

\subsection{Visualisierung Regional Datenbank}

Im Teilprojekt „Regional Foresight“ wurde aus verschiedenen Quellen eine Datenbank zur regionalen Faktorausstattung und zum regionalen Innovationssystem der Projektregion zusammengestellt. Diverse Indikatoren (Stand April 2020: 50 Indikatoren) wurden anschließend gruppiert. Verteilt nach den zur Projektregion gehörenden zehn Landkreisen wurden die Indikatoren für die folgenden Bereiche eingeteilt:

- Ökonomie

- Demografie

- Infrastruktur und Verkehr

- Bildungswesen

- Gesundheitswesen

- Fläche und Wohnen

- Kultur und öffentlicher Raum

- Regionales Innovationssystem

Die Daten wurden für die Jahre 2005 bis 2018 zusammengetragen und müssen jährlich ergänzt werden. Zur Vergleichbarkeit werden, neben den Daten der Landkreise, auch die von Brandenburg, Sachsen und Deutschland erfasst, sofern dies möglich ist. Die im Teilprojekt als „Regional Datenbank“ benannte Datenbank liegt als Microsoft Excel Datei vor. Innerhalb der Microsoft Excel Datei ist eine Übersichtlichkeit nicht gegeben und die erhobenen Daten können so nicht der breiten Öffentlichkeit zugänglich gemacht werden. Daher ist angedacht, die Daten online zu visualisieren und damit zu veröffentlichen. Dafür soll in der digitalen Plattform eine Oberfläche bereitgestellt werden, in der Nutzer:innen die passenden Daten wählen und in Diagrammen anzeigen lassen können. Dabei sollen die Nutzer:innen Auswahlmöglichkeiten zu Landkreisen, Jahren, thematischen Schwerpunkten sowie konkreten Indikatoren erhalten und somit genau die Daten anzeigen lassen können, die von Interesse sind. Ziel ist es, dass die Nutzer:innen nach wenigen Klicks bereits erste Ergebnisse bzw. Diagramme angezeigt bekommen, um den Zugang so leicht wie möglich zu gestalten. Die Indikatoren werden dabei in einer Baumstruktur innerhalb der Sektoren gegliedert.

Es wurde vereinbart, die Visualisierung in einer eigenständigen Anwendung zu implementieren. Dafür soll eine eigene Datenbank entwickelt sowie, darauf aufbauend, die Visualisierung als Webanwendung umgesetzt werden. Die Wahl der Software ist hierbei relativ frei, da am Ende ein virtueller Server stehen wird, dessen Inhalte in die digitale 
Plattform, z.B. per iframe oder ähnlichem, eingebunden werden. Es wurde ein vorläufiges Konzept erarbeitet, wie die gesammelten Daten visuell aufbereitet werden sollen.

In Tabelle 3 sind die für die programmtechnische Umsetzung notwendigen Arbeitspakete mit Bemerkungen aufgelistet.

\begin{tabular}{|c|c|}
\hline Arbeitspaket & Bemerkung \\
\hline \multicolumn{2}{|r|}{ Backend/Datenbank } \\
\hline $\begin{array}{l}\text { Datenbank } \\
\text { auswählen }\end{array}$ & Auswahl, welches Datenbankmanagementsystem (DBMS) am besten geeignet ist. \\
\hline $\begin{array}{l}\text { Datenbank- } \\
\text { Schema } \\
\text { erstellen }\end{array}$ & $\begin{array}{l}\text { Ein vollständiges Datenbank-Schema erstellen, welches alle Indikatoren und } \\
\text { Funktionen beinhaltet und weitgehend normalisiert ist. }\end{array}$ \\
\hline $\begin{array}{l}\text { Daten } \\
\text { importieren }\end{array}$ & $\begin{array}{l}\text { Die in Excel-Sheets vorliegenden Daten müssen in die Datenbank migriert werden. } \\
\text { Dies muss ebenfalls replizierbar sein, wenn neue Daten gegeben sind. }\end{array}$ \\
\hline \multicolumn{2}{|r|}{ Frontend/Website } \\
\hline $\begin{array}{l}\text { Software } \\
\text { auswählen }\end{array}$ & $\begin{array}{l}\text { Auswahl des Webservers, der Programmiersprache und des Frameworks für die } \\
\text { Präsentation der Daten. }\end{array}$ \\
\hline $\begin{array}{l}\text { Datenauswahl } \\
\text { umsetzen }\end{array}$ & $\begin{array}{l}\text { Nutzer:innen sollen die passenden Daten auswählen können. Dabei wird im ersten } \\
\text { Schritt die Gruppe der Indikatoren gewählt und anschließend der gewünschte Indikator. } \\
\text { Dies sind die Pflichtauswahlmöglichkeiten. Zusätzlich können die Nutzer:innen die } \\
\text { Ergebnisse noch nach Jahren, Landkreisen und mit einem Vergleich des } \\
\text { Bundesdurchschnittes filtern. Die angezeigten Diagrammtypen variieren je nach Filter. }\end{array}$ \\
\hline $\begin{array}{l}\text { Diagramme } \\
\text { erstellen }\end{array}$ & $\begin{array}{l}\text { Für die Darstellung der Indikatoren sollen dynamische Diagramme zur Laufzeit erstellt } \\
\text { werden, die auf den von den Nutzer:innnen gewählten Parametern basieren. Dabei } \\
\text { variieren die angezeigten Diagrammtypen automatisch anhand der Parameter. Je nach } \\
\text { Auswahl sollen Linien-, Balken-, Spinnennetz- oder Kreisdiagramme angezeigt } \\
\text { werden. Zusätzlich soll eine Karte angezeigt werden können, in welcher die Landkreise } \\
\text { eingefärbt werden. }\end{array}$ \\
\hline
\end{tabular}

Tabelle 2: Arbeitspakete der Visualisierung der Regional DB.

Damit der Programmcode zentral gespeichert und wiederverwendet werden kann, ist in der BTU Cottbus-Senftenberg im GitLab ${ }^{16}$ ein Repository eingerichtet. Das GitLab kann auch benutzt werden, um Fehlerberichte oder Anforderungen an die Entwickler:innen zu übermitteln, die dann in einer Liste für alle sichtbar sind. Für die Umsetzung wird ein Server an der TH Wildau bereitgestellt. Die Abbildung 5 zeigt den aktuellen Stand der Umsetzung.

\footnotetext{
${ }^{16}$ GitLab ist eine Webanwendung, die der Versionsverwaltung von Quellcode dient. Somit wird sichergestellt, dass Programmierer:innen denselben Stand des Quellcodes nutzen.
} 


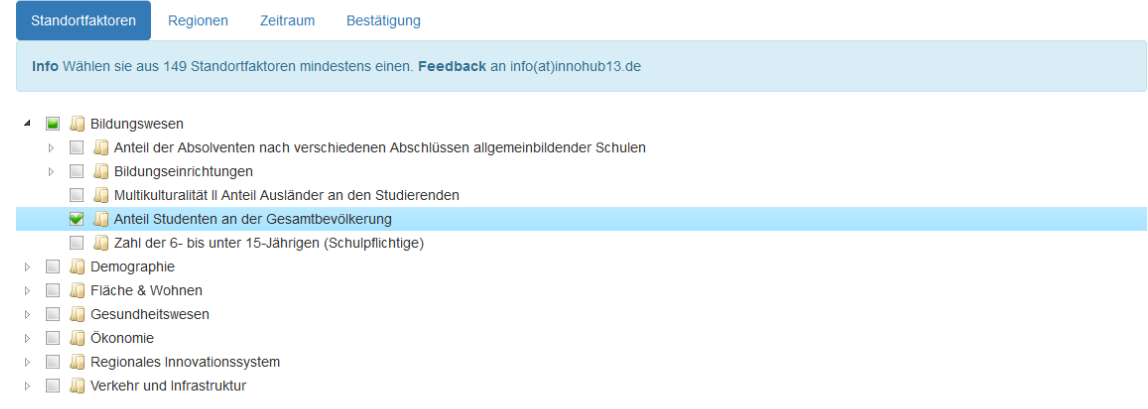

Anteil Studenten an der Gesamtbevölkerung

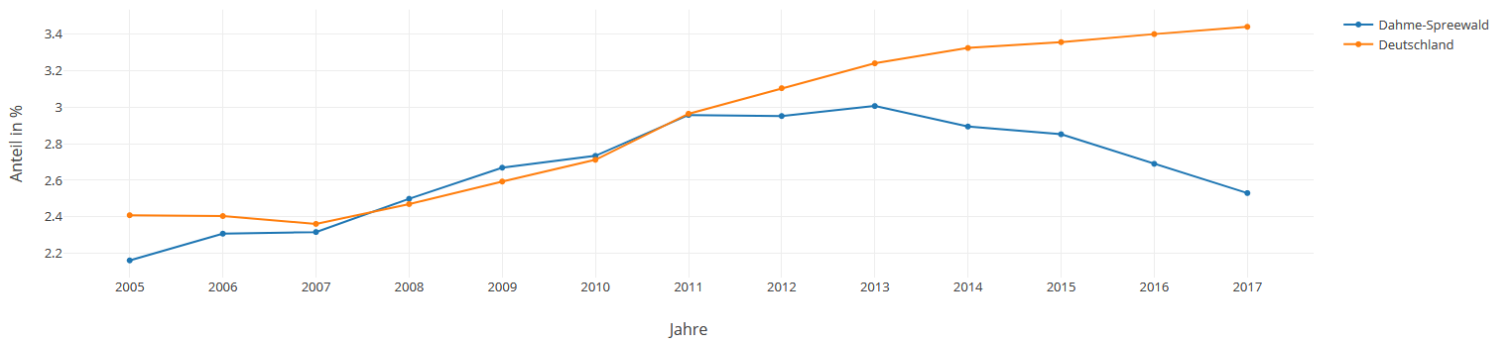

Abbildung 5: Screenshot Stand der Arbeit Visualisierung Regional DB (Colv 2020).

Durch den Screenshot wird verdeutlicht, dass eine hierarchische Baumstruktur der Indikatoren gegeben ist und in der Datenbank abgelegt sowie in der Benutzeroberfläche abgebildet werden muss.

\subsection{InnoRadar}

Ein Bestandteil der digitalen Plattform wird das sogenannte InnoRadar werden. Das InnoRadar ist ein Technologie-Radar, welches die Methoden, Technologien und Geräte der im Innovation Hub 13 verorteten Institute komprimiert präsentiert. Dabei werden die Transfersteckbriefe als Grundlage genommen und jeder Einzelne als ein Datenpunkt - ein sogenannter Blip - abgebildet. Aus Gründen der Übersichtlichkeit - aktuell (Juli 2021) sind 66 Transfersteckbriefe vorhanden - werden drei Radare entstehen. Jeweils eines für die Bereiche digitale Integration, Leichtbau und Life Sciences. Eine Darstellung aller Transfersteckbriefe in einem Radar würde die Nutzer:innen subjektiv überfordern. Zudem würden vor allem in dem innersten Ring Darstellungsprobleme entstehen.

Das InnoRadar ist eine Adaption des XT Tech Radars (XT Rechradar 2018), welches wiederum auf dem Thoughtworks Technology Radar (Thoughtworks 2014) basiert. Das vorhandene Open Source Technologie Radar wurde für die Zwecke des Innovation Hub 13 weiterentwickelt und angepasst. Auf dem Screenshot in Abbildung 6 ist das Radar des Bereiches Life Sciences zu erkennen. 


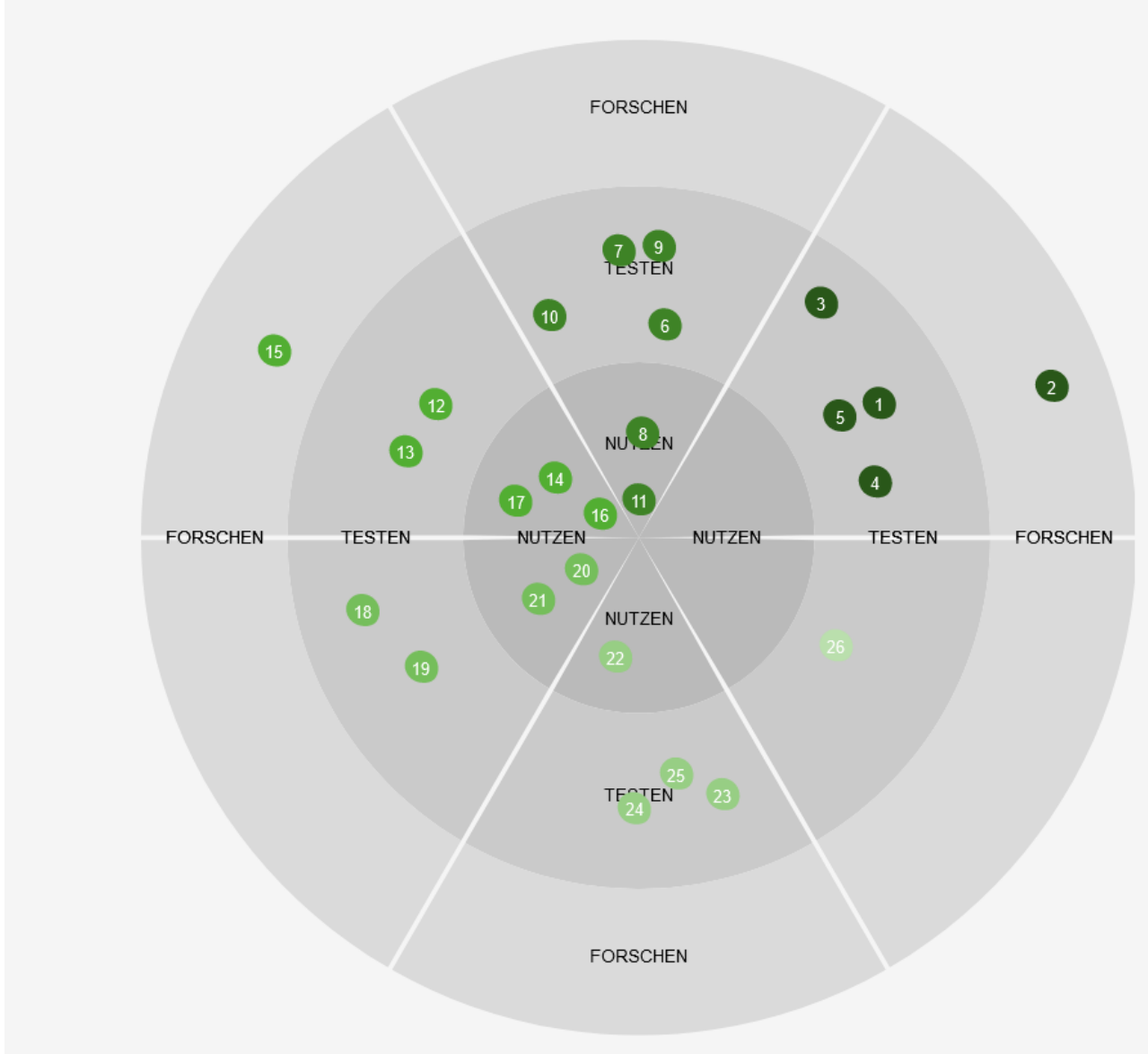

Abbildung 6: Screenshot InnoRadar für den Bereich Life Sciences (Jacob und Berndt 2020).

Der Screenshot zeigt einen großen Teil der Funktionalität des InnoRadars. Das Radar wird exemplarisch für alle drei Radare beschrieben, da die anderen beiden identisch aufgebaut sind. Das beispielhafte Radar des Bereiches Life Sciences ist in sechs Unterkategorien eingeteilt, damit eine Übersicht nach Technologiebereichen möglich ist. Somit ist ein zweiter Clustervorgang - nach der Unterteilung in die drei Hauptgebiete - der Transfersteckbriefe möglich. Zur Darstellung der sechs Unterkategorien wird das $360^{\circ}$ umfassende Radar in sechs Segmente à $60^{\circ}$ unterteilt. Ganz trennscharf ist die Einteilung nicht, da der Inhalt der jeweiligen Bereiche auch anderen Segmenten zugeordnet werden kann. Trotzdem wird eine Grundordnung der technologischen Treiber erzeugt.

Eine weitere Unterteilung der Transfersteckbriefe erfolgt über die Zuordnung in sogenannte Ringe. Die Ringe des Radars beschreiben den Reifegrad der jeweiligen Technologie. Gemäß der Metapher eines Radars steigt der Reifegrad mit der Annäherung an das Zentrum. Dabei geben die einzelnen Ringe eine Bewertung des Technology Readiness Levels (TRL) an. TRLs sind ein systematisches Metrik- und Messsystem, das die Bewertung des Reifegrads einer bestimmten Technologie sowie den konsistenten Vergleich des Reifegrads verschiedener Technologietypen ermöglicht. Dabei werden neun Unterteilungen von Grundlagenforschung (Level 1) bis zum fertig eingesetzten System (Level 9) vorgenommen (Mankins 1995: 28 ff.). Da eine Darstellung von neun Ringen, 
welche für die neun TRLs stehen, in einem Radar unübersichtlich ist und zudem eine Darstellung der Blips aufgeteilt auf neun Ringe eine Platzproblematik verursacht, werden die Level in drei Ringe aufgeteilt. Dabei wird die Unterteilung der Level nach dem 2019 von Berndt und Mietzner vorgestellten Schema vorgenommen. Diese haben eine Einteilung in die drei Unterbereiche „Nutzen“, „Testen“ und „Forschen“ vollzogen. Die schematische Darstellung in Abbildung 7 zeigt die einzelnen Stufen in Anlehnung an diese Einteilung:

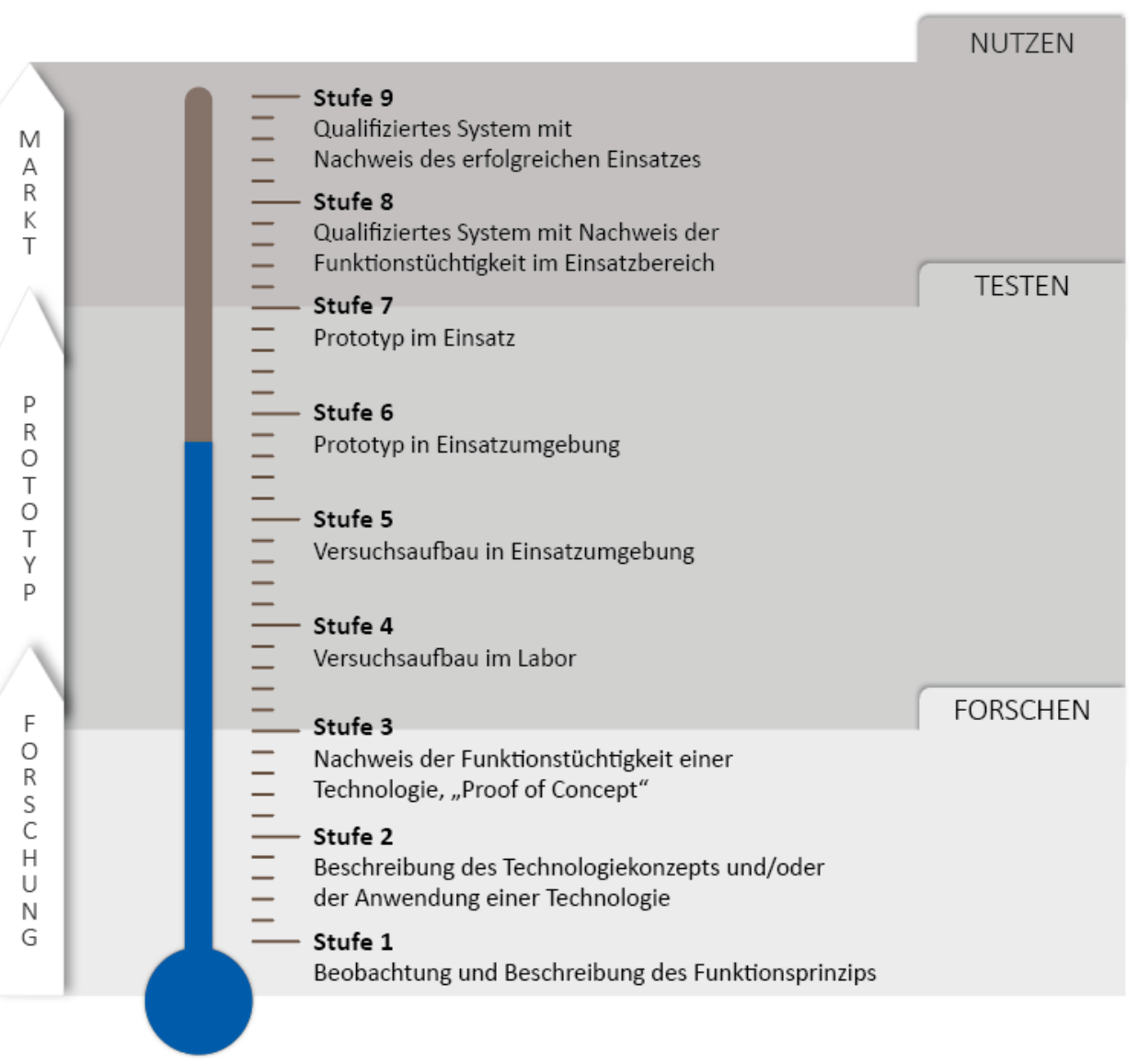

Abbildung 7: Einteilung Technology Readiness Level in drei Level (Berndt und Mietzner 2019: 91).

Analog zu dem Schema werden die Stufen 8 sowie 9 dem innersten Ring des Radars zugeordnet, die Stufen 4 bis 7 dem mittleren Ring und die ersten drei Stufen dem äußersten Ring. Aufgrund der Einteilung der Transfersteckbriefe in Technologiebereich, Unterkategorie und Reifegrad ergibt sich ein dreidimensionales Clustering. Die Einteilung erfolgt durch die Transferscouts und ist vor allem in Bezug auf den Reifegrad in regelmäßigen Abständen zu überprüfen.

Für die benutzerfreundliche Bedienung sind weitere Funktionen implementiert, u.a. Filtermöglichkeiten. Dabei ist es den Nutzer:innen möglich, die angezeigten Transfersteckbriefe nach den beteiligten Instituten zu filtern. Ebenso ist eine Filterung umgesetzt, die es erlaubt, die Blips anzuzeigen, die ein Patent besitzen oder die keines 
nachweisen können. Damit die Benutzer:innen das bevorzugte Fachgebiet selektieren können und eine bessere Vorauswahl angezeigt wird, ist eine Zoom-Funktion auf ein Segment implementiert. Dadurch wird ausschließlich das gewählte Segment und die in diesem verorteten Transfersteckbriefe angezeigt. Eine weitere Filterung innerhalb der Segmente ist möglich.

Neben der filterbaren Übersicht aller Transfersteckbriefe können weitere Details zu den vorgestellten Technologien und Methoden angezeigt werden. Basis sind alle Daten, die im Transfersteckbrief verortet sind. Im Detail sind dies der Status, der Hintergrund, eine Beschreibung der Technologie bzw. Methode, die Vorteile, Anwendungsmöglichkeiten, eine Kontaktperson aus dem Innovation Hub 13, ein Fachkontakt und eine Downloadmöglichkeit des dazugehörigen Transfersteckbriefs. Damit die Benutzer:innen ein Verständnis für das InnoRadar und die Verwendung erhalten, ist ein kleines Handbuch verfügbar. Das Handbuch zeigt den Nutzen des Radars und auch, wie alle Bestandteile zu interpretieren sind.

Ein wichtiger Punkt, der zu bedenken ist, ist die weitere Pflege der Inhalte des InnoRadars. Beispielhafte Szenarien für eine Anpassung sind geänderte Transfersteckbriefe, neue Transfersteckbriefe und eine neue Einsortierung der vorhandenen TSB. Da dies wahrscheinlich häufiger durchgeführt werden muss, ist ein Bestreben zu einer maximal möglichen Automatisierung gegeben. Um dies zu gewährleisten, ist ein neuer Parser programmiert worden, der automatisch aus einer CSV-Datei die passenden Dateien für das InnoRadar erstellt. Der Parser basiert auf der Programmiersprache Python und ist über die Windows PowerShell aufrufbar. Die CSV-Datei kann mit einem Tabellenkalkulationsprogramm, wie beispielsweise Microsoft Excel, bearbeitet werden und beinhaltet den vollständigen Inhalt und die Zuordnung aller Blips. Dadurch kann auch eine Person ohne Programmierkenntnisse die Datei bearbeiten und das InnoRadar aktualisieren. Die erzeugten Dateien müssen in der Folge die vorhandenen Dateien auf dem Server ersetzen.

\subsection{Recommendation Engine}

Für die bessere Suche auf der digitalen Plattform soll eine Recommendation Engine (RE) entwickelt werden, die es ermöglicht, einen inhaltlichen Vergleich mehrerer Datensätze, wie beispielsweise unterschiedlicher Forschungsaktivitäten (an Hochschulen, Unternehmen etc.), durchzuführen. Nach ausgewählten Suchszenarien sollen komplexe Probleme des Vergleichs zwischen zwei Institutionen gelöst werden. Für die Suche nach Forschungspartner:innen soll die RE thematisch verwandte, potenzielle Partner:innen vorschlagen. Ausgangspunkt für einen aufwändigen Vergleich (der sich über mehrere Eigenschaften erstreckt) sind atomare Ähnlichkeitsbedingungen bei ausgewählten Eigenschaften zweier Einrichtungen. Als Resultat des sogenannten Matchings soll die Recommendation Engine gewichtete Suchergebnisse zu bestimmten Szenarien bzw. Anwendungsfällen liefern, die eine maximale Übereinstimmung $\mathrm{zu}$ den eingegebenen 
Daten liefern. Beispielhafte Anwendungsfälle (Use Cases) für den Einsatz der Engine werden in der Folge beschrieben:

- Use Case Hochschule: Der Use Case ist auf die Hochschulen ausgerichtet. Mitarbeiter:innen der Hochschule sollen ihre Projektidee als Freitext eingeben können (z.B. ein vorhandenes Abstract), und die Engine soll passende Projektausschreibungen sowie Industriepartner ausgeben. Dadurch wird die Suche für die Hochschulen vereinfacht und eine Projektanbahnung erleichtert.

- Use Case Unternehmen I: Bei diesem Anwendungsfall sollen Industriebeteiligte ihre technischen Problemstellungen in Textform angeben können. Die RE gibt die passenden Lehrstühle sowie die Fördermöglichkeiten durch Bund und Land aus. Diese Funktion soll dafür sorgen, dass die Hemmschwelle für die Wirtschaft abgebaut wird, sich mit den Hochschulen zu verbinden. Des Weiteren werden die Suche nach den richtigen Partner:innen und Fördermöglichkeiten vereinfacht. Das sorgt u.a. für einen schnelleren Projektbeginn.

- Use Case Unternehmen II: Wenn ein Unternehmen ein neues Produkt entwickelt hat, dann benötigt es die Möglichkeit, es ausgiebig in diversen Szenarien zu testen. In der Recommendation Engine soll das Unternehmen die Informationen zu dem Produkt in Freitext eingeben können und die Algorithmen geben passende Testfelder aus.

- Use Case Land: Um Ausschreibungen des Landes gezielt zu adressieren, soll das System bei Eingabe von strategischen Zielen geeignete Partner:innen aus Industrie und Wissenschaft identifizieren. Diese können dann direkt auf die Ausschreibungen hingewiesen werden. So wird die Chance erhöht, dass passende Angebote für die Gesuche erhalten werden.

- Use Case Studierende: Studierende sollen die Möglichkeit bekommen, ihre Fähigkeiten, ihr Vorwissen und ihre Interessen einzugeben. Je nach Intention der Studierenden soll der Algorithmus passende Abschlussarbeits- oder Stellenangebote präsentieren. Das führt zu einer vereinfachten Suche für die Studierenden. Es sorgt im Gegenzug direkt dafür, dass die Unternehmen passende Interessierte erhalten, die sonst nicht auf die Idee gekommen wären, sich bei ihnen zu bewerben.

- Use Case Fördermittelgeber: Um für Förderprogramme die richtigen Adressaten zu finden, sollen Fördermittelgeber die Engine verwenden können. Sie sollen das Förderprogramm in Textform eingeben können und anschließend von dem Algorithmus die passenden Unternehmen und Lehrstühle ausgegeben bekommen. Diese können dann konkret über das jeweilige Förderprogramm informiert werden.

Diese Beispielszenarien bzw. Anwendungsfälle zeigen das große Spektrum, welches die Recommendation Engine langfristig ermöglichen und bereitstellen soll. Die Engine ist in der Entwicklung, und es werden nach und nach Use Cases umgesetzt werden. Da die Eingaben und die Datenbasis oftmals aus Texten bestehen, ist ein einfacher Vergleich von Werten problematisch. Daher sollen Machine Learning Algorithmen sowie semantische 
Vergleiche bei den Auswertungen und Empfehlungen helfen. Aktuell ist eine interne Alpha-Version entstanden, die bereits einen geringen Funktionsumfang beinhaltet. Beispielsweise ist eine Eingabe von Organisationen (Unternehmen/ Hochschulen etc.), Personen, Projekten, Projektergebnissen sowie Ausstattungen von Laboren möglich. Auch eine Ausgabe dieser Entitäten und eine Erstellung von Transfersteckbriefen ist umgesetzt. Die Daten für die Steckbriefe werden aktuell per Knopfdruck via Datenbankzugriff auf die EGroupware erstellt. Geplant ist eine automatische Erstellung aus den Daten, die per API von AtriFlow generiert werden. ${ }^{17}$ Zudem wurden weitere Datenbestände sondiert und teilweise integriert. Exemplarisch sind über ein Web-Interface der BTU CottbusSenftenberg aktuelle Daten zu Förderprogrammen zugänglich. Ein erster Prototyp, der die Daten herunterlädt und danach in ein einfaches Format für die Weiterverarbeitung bringt, wurde entwickelt. Damit können die Daten zu Drittmittelprojekten an der BTU Cottbus Senftenberg genutzt werden. Der Screenshot in Abbildung 8 zeigt die Alpha-Version der Recommendation Engine.
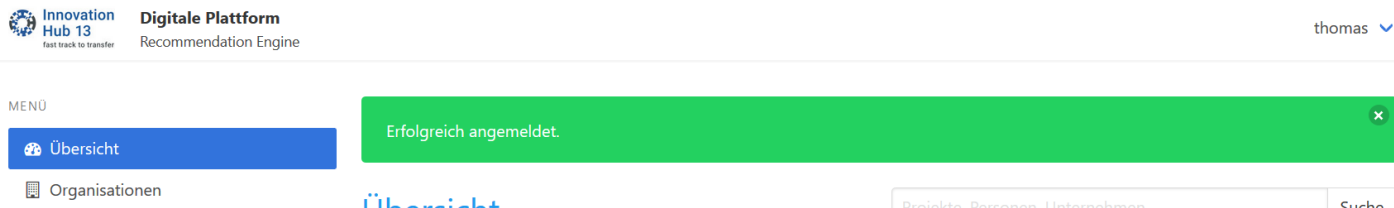

Übersicht

D Personen
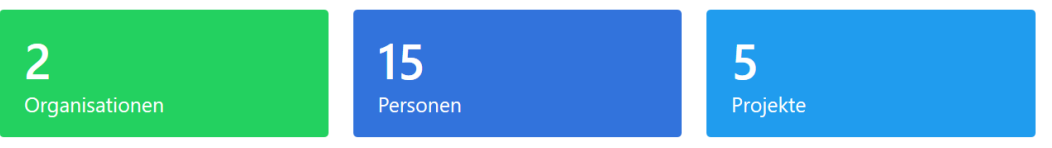

目 Transfersteckbriefe

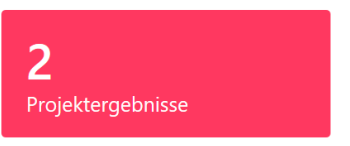

5

Ausstattungen

\section{3}

旨 Transferfragebögen

Abbildung 8: Screenshot Alpha-Version Recommendation Engine Startseite

(Städing und Huber 2018).

Der Screenshot beinhaltet die Startseite der ersten Version, welche eine Suche und eine Filterung der oben genannten Entitäten ermöglicht. Das Matching via Machine Learning ist nicht integriert. Die Plattform ist mithilfe des Webframework Ruby on Rails ${ }^{18}$ realisiert, welches auf der Programmiersprache Ruby basiert. Für die Datenhaltung kommt eine PostgreSQL-Datenbank zum Einsatz. Zur Veranschaulichung der Funktionalität wurde prototypisch eine Umsetzung der Suche mittels Elasticsearch ${ }^{19}$ durchgeführt und in eine

\footnotetext{
${ }^{17}$ Siehe Kapitel 3 Datenerhaltung der digitalen Plattform des Innovation Hub 13.

${ }^{18}$ Ruby on Rails (RoR) ist ein Web-Anwendungsframework, das auf Ruby, einer objektorientierten Skriptsprache, basiert. RoR ist auf Ruby aufgebaut und nutzt eine model-view-controller (MVC)-Architektur, bei der jede Webanfrage durch den Client zu einem Aufruf einer Methode in einem Controller führt. Der Controller wiederum verwendet ein Modell, um Datenbankzugriffe durchzuführen und schließlich eine Ansicht, z.B. den Text einer Webseite, als Antwort zurückgibt (An et al. 2009: 590 ).

${ }^{19}$ Elasticsearch ist eine Open Source Volltextsuchmaschine, die auf Apache Lucene basiert. Sie durchsucht nicht die gegebenen Dokumente bzw. Daten, sondern erstellt im Vorlauf für diese einen Index, der essentielle
} 
lokale Version integriert. Im Screenshot in Abbildung 9 wird das Ergebnis eines Matchingvorgangs gezeigt.

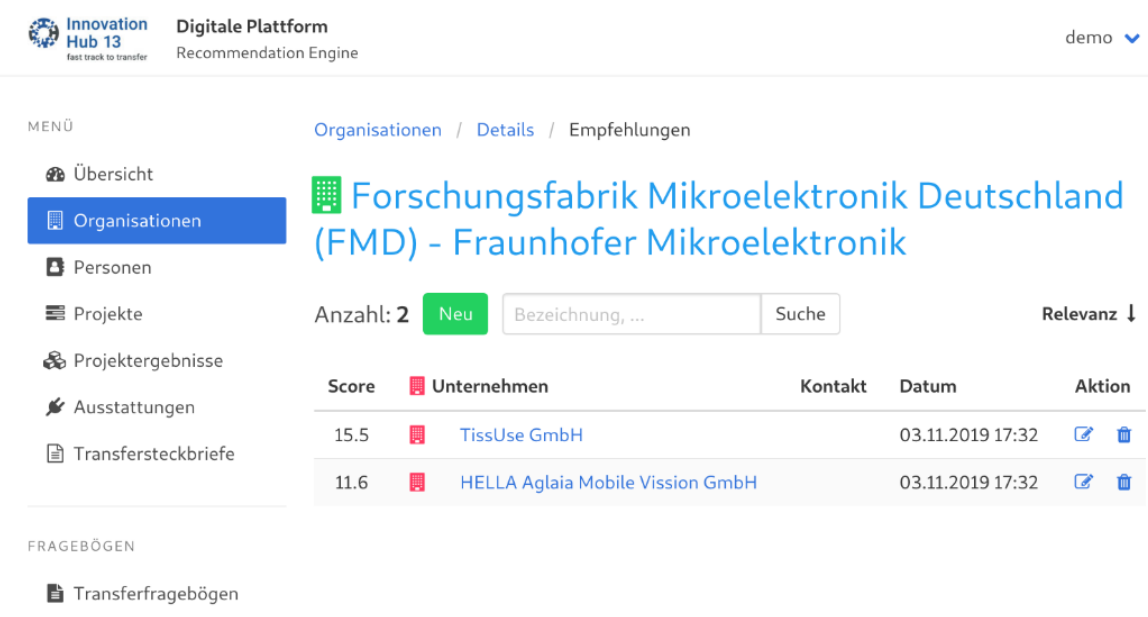

Abbildung 9: Prototypische Darstellung der Ergebnisse der Recommendation Engine (Städing 2019: 75).

Im Screenshot sind Empfehlungen für passende Partner:innen der Forschungsfabrik Mikroelektronik Deutschland des Fraunhofer-Verbund Mikroelektronik zu sehen. Durch den Score-Wert wird die Relevanz der angezeigten Unternehmen angezeigt und eine Gewichtung geschaffen. Das Matching funktioniert in engen Bedingungen, aber es verdeutlicht, dass die Recommendation Engine prototypisch funktioniert.

\subsection{Entitäten der Institute}

Die digitale Plattform soll auch der Darstellung der Entitäten dienen, die an den beteiligten Instituten gegeben sind. Unter anderem sollen vorhandene Geräte, Labore, Projekte, Personen und Showrooms präsentiert werden. Diese sollen abgerufen und weitere Informationen zu diesen gegeben werden können. Mithilfe von Filter- und Suchfunktionen sollen die Entitäten angezeigt werden, die für Nutzer:innen interessant sind. Eine einheitliche sowie konsequente Verschlagwortung ist notwendig, um die besten Ergebnisse für die Nutzer:innen zu präsentieren. Durch die passenden Kontaktdaten für die Verantwortlichen der Entitäten soll ein niedrigschwelliger Zugang zwischen Nutzer:innen und den Instituten bzw. dem Innovation Hub 13 entstehen. Die Daten und Dateien (z.B. Bilder und Datenblätter) für die Entitäten sollen nicht in der digitalen Plattform verwaltet sowie gespeichert werden, sondern in der Plattform von Atrineo. ${ }^{20}$ Dadurch werden Redundanzen vermieden und eine Datenpflege erleichtert. Der Zugriff soll mithilfe der bereitgestellten API von Atriflow funktionieren.

Daten beinhaltet. Bei Suchanfragen, wird der Index durchsucht, was eine deutliche Performance Steigerung zur Folge hat (1\&1 IONOS SE 2019).

${ }^{20}$ Siehe Kapitel 3 Datenhaltung der digitalen Plattform des Innovation Hub 13. 


\section{Wireframing}

Für die Veranschaulichung und das grobe Verständnis der Nutzung der digitalen Plattform sind Wireframes entstanden. Diese wurden erstellt während der Konzeptionierung und vor der ersten, programmatischen Erstellung des Prototyps der digitalen Plattform. Ein Wireframe ist eine Art Blaupause einer Webseite bzw. Plattform. Dabei entsteht ein zweidimensionaler skelettartiger Umriss der Plattform. Wireframes bieten einen klaren Überblick über die Seitenstruktur, das Layout, die Informationsarchitektur, den Benutzerfluss, die Funktionalität und das beabsichtigte Verhalten. Da ein Wireframe in der Regel das anfängliche Produktkonzept darstellt, sind Styling, Farbe und Grafiken auf ein Minimum beschränkt. In der Praxis wird Wireframing hauptsächlich von UXDesigner:innen ${ }^{21}$ verwendet und dient der Platzierung der Informationen und Elementen der Webseite oder Plattform (Hannah o. J.).

Die entstandenen Wireframes bilden nicht den gesamten Funktionsumfang der digitalen Plattform $\mathrm{ab}$ und sind erste Entwürfe. Die Wireframes sind über die Seite https://app.diagrams.net/ entstanden, die auf dem Open Source Projekt draw.io basiert (JGraph 2017). Der Screenshot in Abbildung 10 zeigt den aktuellen Stand des Wireframes der Startseite der digitalen Plattform.

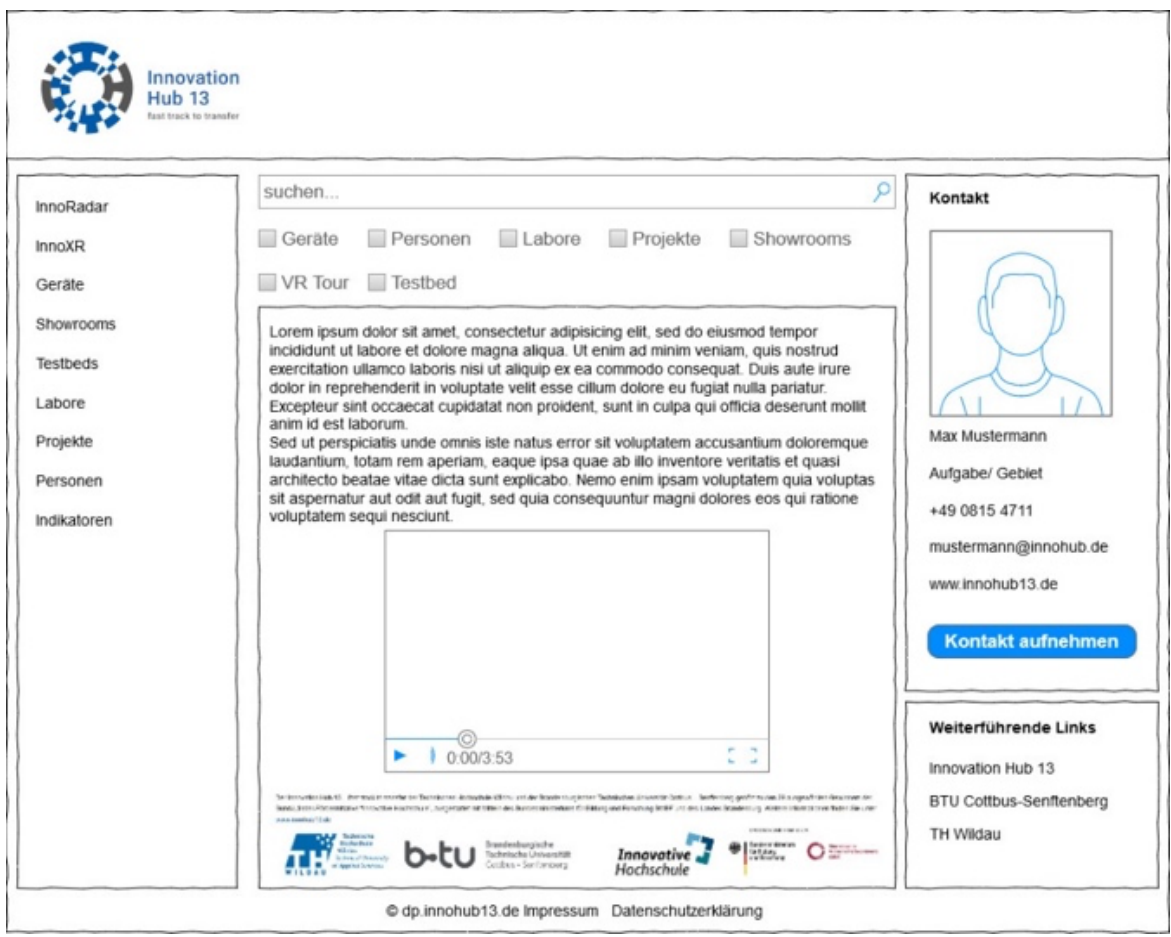

Abbildung 10: Wireframe-Entwurf der Startseite der digitalen Plattform.

${ }^{21}$ UX steht für User Experience und beschreibt die Erfahrung, die Benutzer:innen erleben, wenn sie eine Webseite oder ähnliches besuchen. 
Die digitale Plattform soll aus fünf Unterbereichen bestehen: Ein Header, eine linke wie rechte Sidebar, ein Content-Bereich und ein Footer.

- Header-Bereich: Der Kopfbereich beschreibt den oberen Teil einer Webseite. Dieser beinhaltet das Logo und ggf. ein Navigationsmenü im rechten Bereich. Der Bereich wird statisch sein und sich während der Nutzung nicht verändern.

- Linke Sidebar: In der linken Seitenleiste wird die Hauptnavigation integriert. Über diese Navigation werden die einzelnen Seiten erreichbar sein.

- Rechte Sidebar: In der rechten Seitenleiste werden Kontaktmöglichkeiten und weiterführende Links präsentiert. Die Nutzer:innen sollen somit den für den jeweiligen Bereich passenden Kontakt direkt angezeigt bekommen und dazu animiert werden, diesen zu kontaktieren.

- Content-Bereich: Im Contentbereich, der sich zentral auf der Seite befindet, soll der eigentliche Inhalt angezeigt werden. Je nach Seite variiert der Inhalt stark. Der Inhalt soll dynamisch präsentiert werden und teilweise Interaktionen mit den Nutzer:innen ermöglichen. Neben dem reinen Inhalt soll die Möglichkeit gegeben werden, dass Content gesucht und gefiltert werden kann. In diesem Entwurf wird diese Möglichkeit im ContentBereich bereitgestellt.

- Footer-Bereich: Der Fußbereich der digitalen Plattform soll statisch sein und einen Copyright-Vermerk, einen Link zum Impressum sowie zur Datenschutzerklärung beinhalten.

Die entstandenen prototypischen Wireframes zeigen eine Möglichkeit, die digitale Plattform des Innovation Hub 13 umzusetzen. Anhand dieser soll die schrittweise Umsetzung der Plattform durchgeführt und sukzessive die einzelnen Bausteine integriert werden.

\section{Datenschutz}

Bei der digitalen Plattform werden, neben allgemeinen Daten zu Unternehmen und Hochschulen, personenbezogene Daten genutzt, verarbeitet und präsentiert. Aus diesem Grund ist eine Betrachtung der Datenschutzrichtlinien gemäß der 2018 in Kraft getretenen europäischen Datenschutz-Grundverordnung (DSVGO) notwendig.

Der Innovation Hub 13 versteht sich als innovative Institution mit dem Ziel, Wege aufzuzeigen, wie die Einhaltung des Datenschutzes umgesetzt werden kann, ohne funktionale wie ästhetische Einbußen zu verzeichnen. Daher wurde eine erhebliche Einarbeitung in die Thematik durchgeführt. Nach dieser wurde festgestellt, welche Daten in den Teilprojekten erhoben werden. Daraus wurden die Verarbeitungstätigkeiten und das Verarbeitungsverzeichnis abgeleitet. Da die Daten projektpartner-übergreifend verarbeitet werden müssen (z.B. personenbezogene Daten in der Transferplattform), musste in der Folge ein Vertrag für die gemeinsame Verantwortlichkeit erstellt werden. Dieser wurde von den einzelnen Institutionen nach mehreren Änderungen bestätigt und unterschrieben. Auf Basis dieses Vertrages wurde ein Informationsschreiben bzw. eine Einverständniserklärung erstellt, welche u.a. die interviewten Personen vorgelegt 
bekommen und unterschreiben können. Zur Einpflege wie Bearbeitung personenbezogener Daten in AtriFlow muss eine unterschriebene Einverständniserklärung (analog oder digital) vorliegen. Sollte eine Person die Einverständniserklärung widerrufen, wird der Datensatz aus der Datenhaltung in AtriFlow entfernt und damit auch aus den Anzeigen der digitalen Plattform. ${ }^{22}$ Anders verhält es sich mit der Verwaltung von Nutzerkonten für die digitale Plattform. Bei der Anmeldung muss eine Einverständniserklärung eingeholt werden. Dies wird in der Praxis durch eine Bestätigung der Datenschutzrichtlinien gewährleistet. Ein Prinzip der DSVGO ist Datensparsamkeit. Von daher sollten nur die nötigsten Daten der Nutzer:innen gespeichert werden. Für eine einfache Anmeldung sollte die E-Mail-Adresse genügen. Alle weiteren Angaben sind freiwillig. Neben den personenbezogenen Daten ist ein wichtiger Bestandteil der DSVGO die sichere Übertragung und Verarbeitung der Daten. Daher sind eine verschlüsselte Übertragung der Daten via SSL und eine sichere Speicherung für die Plattform angedacht. Der Datenschutz ist durch die Einführung der DSGVO vereinheitlicht und die Daten von Personen mehr geschützt worden. Dies ist als sehr positiv zu betrachten, aber es bringt neue Herausforderungen mit sich. Jene Herausforderung gilt es zu bestehen und die passenden Maßnahmen zu ergreifen. Dies gilt für den Innovation Hub 13 wie auch explizit für die digitale Plattform.

\section{Zusammenfassung und Ausblick}

Die digitale Plattform wird als digitaler Zwilling des Innovation Hub 13 entwickelt. Dazu sollen alle Bestandteile des Hubs, inkl. der Showrooms und Testfelder, abgebildet werden. Um das zu ermöglichen, sind einige Bausteine der geplanten Plattform prototypisch umgesetzt oder zumindest in der Konzeptionierung. Gleichzeitig soll die digitale Plattform den Mitarbeiter:innen als Werkzeug dienen, um den Transfer zu ermöglichen.

Dieses Working Paper zeigt den Stand der digitalen Plattform (Stand Juni 2020) des Innovation Hub 13 und die konzeptionellen Überlegungen, die zu diesem Stand geführt haben. Neben den bisherigen Arbeiten wurde beschrieben, was eine digitale Plattform auszeichnet und wie diese umgesetzt werden kann. Eine Abgrenzung zu den eingesetzten Softwarelösungen, welche die Mitarbeiter:innen des Innovation Hub 13 bei ihrer Arbeit unterstützen, wurde durchgeführt.

Die Datenhaltung und der Datenzugriff wird zukünftig mithilfe der Transferplattform AtriFlow umgesetzt werden, sodass die Daten nicht redundant eingegeben werden müssen. Es wurden bereits einzelne Bausteine der geplanten Plattform umgesetzt und diese funktionieren aktuell als Standalone-Varianten. Exemplarisch sind das InnoRadar, die Recommendation Engine, die VR-Touren und die Visualisierung der Regional Datenbank zu nennen. Für die Veranschaulichung und das grobe Verständnis der Nutzung der digitalen

\footnotetext{
${ }^{22}$ Dies kann zu Anzeigeproblemen bei Kontaktpersonen führen und muss dementsprechend bedacht werden.
} 
Plattform sind Wireframes erstellt worden. Auch eine Betrachtung des Datenschutzes in Verbindung mit der digitalen Plattform wurde durchgeführt und es wurde abgeschätzt, welche Maßnahmen durchgeführt werden müssen, um den Richtlinien der DatenschutzGrundverordnung zu entsprechen. Es wurden u.a. Einverständniserklärungen, ein Verarbeitungsverzeichnis und ein institutsübergreifender Vertrag für die gemeinsame Verantwortlichkeit erstellt, um dem Datenschutz der personenbezogenen Daten gerecht zu werden. Zwar ist dieses Paper nicht allumfassend, aber es zeigt grob die bisherigen Arbeiten und die Planungen für die Zukunft.Es wurde deutlich, dass bereits einige konzeptionelle und ebenso umsetzende Arbeiten durchgeführt worden sind, aber die Entwicklung der digitalen Plattform weiterhin vorangetrieben werden muss. Für die Fertigstellung der Plattform müssen in Zukunft weitere Schritte umgesetzt werden.

Ein wichtiger nächster Schritt wird die Fertigstellung der Transferplattform AtriFlow sein, damit die Datenhaltung der digitalen Plattform sichergestellt ist. Mit Fertigstellung der AtriFlow-Lösung steht die konkrete Datenbank fest, auf die mit Hilfe der API zugegriffen werden kann. Anschließend werden voraussichtlich nur kleine Änderungen an der Datenbasis durchgeführt werden. Dadurch ist eine weitgehende Plansicherheit gegeben. Zu diesem Zeitpunkt können die endgültigen Imports in AtriFlow durchgeführt werden. Es sind dadurch viele Datensätze für die AtriFlow-Lösung sowie die digitale Plattform gegeben.

Des Weiteren muss die digitale Plattform prototypisch mithilfe des Framework Vue.js umgesetzt werden. Als Basis für die Gestaltung muss das Corporate Design des Innovation $H u b 13$ verwendet werden. Für den strukturellen Aufbau dienen die erstellten Wireframes. In diese Plattform müssen die vorhandenen Bausteine integriert werden. Während der Erstellung und des Betriebs müssen begleitend Tests durchgeführt werden, um sicherzustellen, dass die Funktionen wunschgemäß gegeben sind und es zu keinen Fehlern kommt. Sobald eine fertige digitale Plattform erstellt worden ist, die funktionell, gestalterisch und unter Nutzerfreundlichkeits-Aspekten den Anforderungen genügt, kann ein Launch dieser für die Öffentlichkeit durchgeführt werden. Entwicklung der bisherigen Arbeiten zu schaffen, wird der Wandel in der Folge dargestellt. 


\section{$\underline{\text { Literaturverzeichnis }}$}

1\&1 IONOS SE (2019): Elasticsearch: Die flexible Search Engine. Online verfügbar unter: https://www.ionos.de/digitalguide/server/konfiguration/elasticsearch/, zuletzt aktualisiert am 06.05.2020.

A-Frame (o. J.): Introduction. Online verfügbar unter:

https://aframe.io/docs/1.0.0/introduction/, zuletzt geprüft am 06.05.2020.

An, Jong-hoon; Chaudhuri, Avik; Foster, Jeffrey S. (2009): Static Typing for Ruby on Rails. In: 2009 IEEE/ACM International Conference on Automated Software Engineering, S. 590-594.

Berndt, Marko; Mietzner, Dana (2019): Die Entwicklung eines Technologie Radars für den IT-Mittelstand. In: TH Wildau - Wissenschaftliche Beiträge 2019. Wildau: TH Wildau, S. 87-94.

Colv, Marvin (2020): Aktueller Stand Visualisierung Regional DB, 15.04.2020. Mattermost-Chat on Thomas Jacob.

Hannah, Jaye (o. J.): What Exactly Is Wireframing? A Comprehensive Guide. Online verfügbar unter: https://careerfoundry.com/en/blog/ux-design/what-is-a-wireframe-guide/, zuletzt geprüft am 14.05.2020.

Irvine, Kaitlyn (2017): XR: VR, AR, MR — What's the Difference? Online verfügbar unter: https://www.viget.com/articles/xr-vr-ar-mr-whats-the-difference/, zuletzt geprüft am 06.05.2020.

Jacob, Thomas; Berndt, Marko (2020): InnoRadar - Bereich Life Sciences. Online verfügbar unter: https://radar.innohub13.de/lifesciences/, zuletzt aktualisiert am 2020, zuletzt geprüft am 12.04.2020.

JGraph (2017): GitHub - jgraph/drawio: Source to app.diagrams.net. Online verfügbar unter: https://github.com/jgraph/drawio, zuletzt aktualisiert am 2020, zuletzt geprüft am 14.05.2020.

Koenig, Nico (2019): Vue vs. Angular: Die All-in-One Plattform und der Open Source Baukasten. Online verfügbar unter: https://www.hosteurope.de/blog/vue-vs-angular-dieall-in-one-plattform-und-der-open-source-baukasten/, zuletzt geprüft am 01.07.2020.

Krause, Stefan (2020): Results for js web frameworks benchmark - round 8. Online verfügbar unter: https://rawgit.com/krausest/js-framework-benchmark/master/webdriverts-results/table.html, zuletzt geprüft am 01.07.2020. 
Mankins, John (1995): Technology Readiness Level - A White Paper. Online verfügbar unter: http://www.artemisinnovation.com/images/TRL_White_Paper_2004-Edited.pdf, zuletzt geprüft am 19.07.2021, Washington,D.C.: NASA.

Rambeau, Michael (2020): 2019 JavaScript Rising Stars. Front-end Frameworks. Online verfügbar unter: https://risingstars.js.org/2019/en/\#section-framework, zuletzt aktualisiert am 2020, zuletzt geprüft am 25.05.2020.

Rixecker, Kim (2017): Nach Kritik: Facebook stellt React und andere Open-SourceProjekte unter MIT-Lizenz. Online verfügbar unter: https://t3n.de/news/facebook-opensource-mit-lizenz-react-flow-860796/, zuletzt aktualisiert am 2017, zuletzt geprüft am 30.06.2020.

Rochet, Jean-Charles; Tirole, Jean (2006): Two-sided markets: a progress report. In: The RAND Journal of Economics 37 (3), S. 645-667. DOI: 10.1111/j.17562171.2006.tb00036.x.

Schlemmer, Frederik (2019): Angular, React oder Vue.js? Eine Entscheidungshilfe. Online verfügbar unter: https:/www.adesso.de/de/news/blog/angular-react-oder-vue-jseine-entscheidungshilfe.jsp, zuletzt geprüft am 01.07.2020.

Skoczylas, Simon (o. J.): Was unterscheidet React von Frameworks wie Angular und Vue.js? Online verfügbar unter: https://eppleton.de/news/was-unterscheidet-react-vonframeworks-wie-angular-und-vuejs_2019-03-11.html, zuletzt geprüft am 01.07.2020.

Springer, Sebastian (2018): JavaScript Frameworks im Vergleich: Vue vs. Angular vs. React. Online verfügbar unter: https://entwickler.de/online/javascript/vue-angular-reactframework-579849723.html, zuletzt geprüft am 01.07.2020.

Städing, Bertram (2019): Masterarbeit - Entwurf einer Recommendation Engine für das Projekt Innovation Hub 13. Cottbus, BTU Cottbus-Senftenberg.

Städing, Bertram; Huber, Markus (2018): Digitale Plattform - Recommendation Engine. Online verfügbar unter: https://innohub13.informatik.tu-cottbus.de/, zuletzt aktualisiert am 2019, zuletzt geprüft am 05.05.2020

Tappert, Isaak (2020): Angular vs. React vs. Vue.js - JavaScript Frontend Frameworks im Vergleich. Online verfügbar unter: https://tappert-it.de/2020/01/01/angular-react-vuejavascript-frontend-frameworks-vergleich/, zuletzt geprüft am 01.07.2020.

Teklote, Johannes (2019): Open-Source-Lizenzen und was ich damit machen darf. Online verfügbar unter: https://www.adesso.de/de/news/blog/open-source-lizenzen-und-was-ichdamit-machen-darf.jsp, zuletzt geprüft am 30.06.2020. 
Teufel, Marc (2018): Vue.js Tutorial: Einführung in das JavaScript-Framework. Online verfügbar unter: https://entwickler.de/online/javascript/tutorial-vue-js-einfuehrung579851571.html, zuletzt geprüft am 01.07.2020.

Thoughtworks (2014): GitHub - thoughtworks/build-your-own-radar: A library that generates an interactive radar. Online verfügbar unter:

https://github.com/thoughtworks/build-your-own-radar, zuletzt aktualisiert am 2019, zuletzt geprüft am 09.04.2020.

XT Rechradar (2018): GitHub - pagesource/xt-techradar: A Tech Radar to help all eXperience Technologists. Online verfügbar unter: https://github.com/pagesource/xttechradar, zuletzt aktualisiert am 2019, zuletzt geprüft am 09.04.2020. 